Article

\title{
Image Quality Assessment of Digital Image Capturing Devices for Melanoma Detection
}

\author{
Bogdan Dugonik $^{1, *}$, Aleksandra Dugonik ${ }^{2}$, Maruška Marovt ${ }^{2}$ (-) and Marjan Golob ${ }^{1}$ \\ 1 Faculty of Electrical Engineering and Computer Science, University of Maribor, 2000 Maribor, Slovenia; \\ marjan.golob@um.si \\ 2 Department of Dermatology, University Medical Centre Maribor, 2000 Maribor, Slovenia; \\ aleksandra.dugonik@ukc-mb.si (A.D.); maruska.marovt@gmail.com (M.M.) \\ * Correspondence: bogdan.dugonik@um.si; Tel.: +386-2-220-7214
}

Received: 24 March 2020; Accepted: 16 April 2020; Published: 21 April 2020

\begin{abstract}
The fast-growing incidence of skin cancer, especially melanoma, is the guiding principle for intense development of various digital image-capturing devices providing easier recognition of melanoma by dermatologists. Handheld and digital dermoscopy, following of mole changes with smartphones and digital analysing of mole images, is based on evaluation of the colours, shape and deep structures in the skin moles. Incorrect colour information of an image, under- or overexposed images, lack of sharpness and low resolution of the images, can lead to melanoma misdiagnosis. The purpose of our study was to determine the colour error in the image according to the given lighting conditions and different camera settings. We focused on measuring the image quality parameters of smartphones and high-resolution cameras to compare them with the results of state-of-the-art dermoscopy device systems. We applied standardised measuring methods. The spatial frequency response method was applied for measuring the sharpness and resolution of the tested camera systems. Colour images with known reference values were captured from the test target, to evaluate colour error as a CIELAB (Commission Internationale de l'Eclairage) $\Delta E_{a b}^{*}$ colour difference as seen by a human observer. The results of our measurements yielded two significant findings. First, all tested cameras produced inaccurate colours when operating in automatic mode, and second, the amount of sharpening was too intensive. These deficiencies can be eliminated through adjusting the camera parameters manually or by image post-production. The presented two-step camera calibration procedure improves the colour accuracy of captured clinical and dermoscopy images significantly.
\end{abstract}

Keywords: dermoscopy; melanoma detection; mole screening; image quality; colour response; spatial frequency response; image resolution; image sharpness

\section{Introduction}

Melanoma is a malignant tumour that arises from melanocytes and involves primarily the skin. Melanomas are usually heavily pigmented but can also be amelanotic. Even small melanomas may tend to metastasise, leading to a relatively unfavourable prognosis, and account for $90 \%$ of skin cancer deaths [1]. Dermoscopy was added to the clinical examination in the nineties as a noninvasive tool to improve diagnostic ability [2]. Dermoscopy, or dermatoscopy, uses a handheld microscope called a dermoscope or dermoscope, that is equipped with a magnification lens and a light source. It facilitates visualisation of subsurface skin structures otherwise not visible to the unaided eye [3]. Today, dermoscopy is an integrative part of the clinical examination, adding significantly to the early detection of skin cancer [2]. Total body photography (TBP) and dermoscopy make it possible to detect a melanoma very early in its evolution, while decreasing the number of unnecessary biopsies and improving recognition of atypical lesions [4,5]. In skin imaging, capturing colour accurately is 
a necessary [6]. Colours for melanin range from black and brown to grey and blue, depending on the location of the melanin within the skin layers, in terms of depth [3]. Even a hard-to-detect hue difference could make a significant difference in melanoma diagnosis [7].

Photographs are invaluable in dermatologic diagnostics, management, research, teaching, and documentation tools [8,9]. Dermatologists specialised in skin cancer use TBP and dermoscopy routinely for melanoma detection [10,11]. However, the use of technological aids for melanoma detection by dermatologists in general practice and primary care physicians has been slow, due to cost and inconvenience [4]. This is changing due to the mobile revolution, as smartphone-based digital cameras and dermoscopes enter the professional market $[4,12]$. Because of the difference in technologies, different imaging devices can produce different results [8,9]. The proliferation of dermatologic imaging has evolved without the benefit of dermatology specific Digital Imaging and Communications in Medicine (DICOM) standards as exist for other medical specialities. The lack of standards for dermatologic imaging undermines the quality and usefulness of skin lesion imaging $[4,8,9]$. In both the medical and public realms, all will benefit from the development of three categories of standards that help to ensure quality (spatial resolution, colour accuracy, clarity of focus), privacy, and interoperability [4]. Therefore, considering the rapid improvements in digital imaging technologies and ubiquity of digital imaging in our society, it is of the utmost importance to standardise the use of digital imaging in dermatology [9].

In our study, we focus on measurement of the most significant image quality parameters, including sharpness, spatial resolution and colour reproduction, recommended as assessment measures for clinical expertise $[8,13,14]$. The standardised quantitative image measurements methods were applied for the image quality parameters analysis. We analysed data measured from images captured with different smartphones or high-resolution cameras used by dermatologists, to compare them with the results of a state-of-the-art digital dermoscopy system.

Spatial resolution and image sharpness are some of the basic and frequently discussed characteristics of digital imaging devices. An imaging system can distinguish fine details of an image. For conventional digital cameras, standards such as ISO 12233 exist [15], which define a reference target and an evaluation method used for measuring both sharpness and resolution. The spatial frequency response (SFR), analogous to the modulation transfer function (MTF) of an optical imaging system, is one of the measurements for analysis of spatial resolution defined in ISO 12233. Resolution and image sharpness are important and frequently discussed features of a digital dermoscopy system, and can be measured [7-11,16,17]. They can be measured by a subjective comparison of images as in [16], or by using a less accurate 1951 USAF resolution test chart [18]. In this research, we used a slanted-edge method of measuring the SFR that is adopted by several international standards, including ISO 12233 and IEEE [19-22]. Resolution and sharpness were measured on images from a reference target as captured, similar to those acquired in everyday dermatological practice (i.e., full-body, close-up and dermoscopy imaging) [23,24]. A micro-test target was designed to measure sharpness and resolution according to the eSFR method for the dermoscopic image. Dermoscopy images were acquired using a handheld dermoscopy device attached to either a smartphone or a high-resolution camera. Results were then compared with the results obtained on a digital dermoscopy system, which is considered as the gold standard.

Colour assessment is essential for the clinical diagnosis of skin cancers [6,25-29]. However, images captured with different cameras and dermoscopes often present problems of accurate colour reproduction [30]. The colours of these images are different from those seen with the naked eye, and usually also vary from camera to camera. The reason for this is that the auto image processing software built into the cameras is usually designed to generate more saturated colours, pleasant to the broadest population of users [31]. However, the wrong colour and too intensive sharpening are likely to produce artefacts or border contour changes, which can result in less effective melanoma screening, longer diagnosis times, and potentially wrong decisions [16]. Since this is not acceptable, the colour accuracy and constancy of cameras must be improved. They can be provided with a colour calibration procedure [18]. The colour calibration is researched broadly in the computer vision 
community, and several methods have been proposed to solve the problem [32-36]. It can be solved either with hardware or software-based calibration algorithms.

In the dermatology context, the authors in [32] used internal camera parameters (e.g., colour gain, camera aperture, camera offset) to calibrate image colours. The calibration procedure is based on 24 patches with known colour properties. The colour transformation matrix is computed between camera corrected $\mathrm{R}^{\prime} \mathrm{G}^{\prime} \mathrm{B}^{\prime}$ values, and a standard device-independent $\mathrm{XYZ}$ colour space, using the values with 24 known colour properties. Grana et al. [33] provide a complete workflow for dermatology image calibration using a Fotofinder video dermoscope, where a process was proposed to correct non-uniform illumination of the instruments. A dermoscope illuminates the central part of the images differently than the edges. The proposed methods perform colour calibration using 24 colour patches from the Greytag Macbeth ColorChecker chart. Quintana et al. [34] proposed another method to increase the accuracy of colour reproduction in dermoscopy imaging, based on exploring the spectral information of the dermoscopy lighting source. The method is devised for RAW images, but a simple solution has been presented for digital cameras producing JPEG images. The authors in [35] developed an automated calibration method for dermoscopy images using the HSV colour system. The colour calibration filters were developed based on hue, saturation and intensity, using a set of 319 dermoscopy images. The critical future of the presented method is that colour calibration can be performed on image content alone. Barata et al. [36] investigated the influence of colour calibration on the performance of melanoma detection. To improve the classification of multisource images, they compared four colour constancy algorithms to estimate the influence of different light source illumination options.

The colour accuracy for all devices in the test remains an issue, mainly when cameras are operating in auto mode. A simple two-step camera calibration procedure was presented to improve the colour accuracy in dermatology imaging, where the white balance and camera exposure are adjusted manually regarding the known lighting conditions.

This paper is structured as follows. Section 1 presents the motivation and an overview. Devices, applied test charts, instruments, and measuring methods are described in Section 2, Materials and Methods. The results are presented, evaluated and commented upon in Section 3. In Section 4, we discuss results and findings, providing adequate guidance to the end-users in the image quality capturing process.

\section{Materials and Methods}

In the study two randomly selected patients were included who underwent a routine dermoscopy examination of the skin moles due to a phenotype with a very high number $(>100)$ of melanocytic nevi, which increased the risk of melanoma appearance.

Both subjects gave their informed consent for inclusion before they participated in the study. The study was conducted in accordance with the Declaration of Helsinki, and the protocol was approved by the Ethics Committee of the University Medical Centre, Maribor (Project identification code UKC-MB-KME-17/20).

Measurements for melanoma detection imaging were captured using a set of smartphone cameras, including iPhone 5s, iPhone 7, iPhone XR (Apple Inc., Cupertino, CA, USA), Samsung S10, (Samsung Electronics Co., Ltd., Seoul, South Korea) and Huawei P10, (Huawei Technologies Co., Ltd., Shenzhen, China); a high-resolution compact digital mirrorless camera, Canon EOS M10; a DSLR Canon EOS 800D camera, (Canon Inc., Tokyo, Japan); and, a Medicam 1000s camera (FotoFinder Systems GmbH, Bad Birnbach, Germany). The DSLR Canon EOS 800D camera and the Medicam 1000s are combined in the FotoFinder system [37], whereas the DSLR Canon EOS 800D camera is used for capturing images in the high-resolution TBP [38]. The list of cameras, sensor resolutions and parameters of applied lenses are given in Table 1.

Figure 1 shows the standardised Imatest ISO 12233:2017 edge spatial frequency response (eSFR) target chart used for the standard image quality parameter measurements [39]. It is fully compliant with the Low Contrast Edge Spatial Frequency Response ISO Standard, and integrates elements of several image quality parameter measurements, including sharpness, lateral chromatic aberrations, white balance, tonal response, colour accuracy, and noise [40]. We created the test target in vector 
format, based on the template of the ISO 12233:2017 eSFR target used for standard image parameter measurements. The vector graphic template for printing was prepared in Adobe Illustrator software (Adobe Inc., San Jose, CA, USA). Sixteen colour patches of known colorimetric values and elements for targeting image orientation were placed in an appropriate position on the chart. A customised target enables measuring a number of parameters (i.e., sharpness and resolution, colour reproduction quality, noise, exposure accuracy, dynamic range and ISO Sensitivity) from a single image. It can be printed in any size that meets the sharpness measurement requirements of digital cameras with built-in high-resolution sensors. The chart dimension of $90 \times 135 \mathrm{~cm}$ allows for analysis of the image sharpness for camera sensors with up to 25 Megapixels of resolution.

Table 1. Cameras applied in the test.

\begin{tabular}{ccc}
\hline Camera Model & Width $\times$ Length $(\mathbf{p x})$ & Camera Lens \\
\hline iPhone 5s & $3264 \times 2448$ & $4.15($ in $35 \mathrm{~mm}: 29 \mathrm{~mm}) \mathrm{f} / 2.2$ \\
iPhone 7 & $4032 \times 3024$ & $3.99($ in $35 \mathrm{~mm}: 29 \mathrm{~mm}) \mathrm{f} / 1.8$ \\
iPhone XR & $4032 \times 3024$ & $4.25($ in $35 \mathrm{~mm}: 29 \mathrm{~mm}) \mathrm{f} / 1.8$ \\
Samsung S10 (SM-G975F) & $4032 \times 3024$ & $6.00($ in $35 \mathrm{~mm}: 26 \mathrm{~mm}) \mathrm{f} / 2.4$ \\
HUAWEI P10 (EVA-L09) & $3968 \times 2976$ & Leica $4.05($ in 35 mm: $27 \mathrm{~mm}) \mathrm{f} / 2.2$ \\
Canon EOS M10 & $5184 \times 3456$ & $30($ EF-M 15-45) f/3.5-6.3 \\
Canon EOS 800D (FotoFinder) & $6000 \times 4000$ & Tokina AT-X M35 PRO DX 2.8 \\
Medicam 1000s (FotoFinder) & $1920 \times 1080$ & - \\
\hline
\end{tabular}

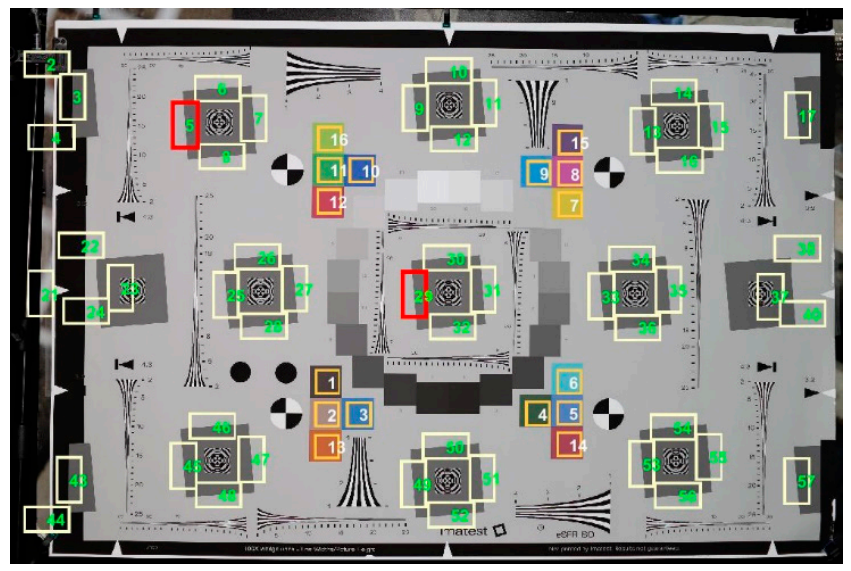

Figure 1. The used edge spatial frequency response (eSFR) ISO test chart target. Sixteen colours are similar to the industry-standard 24-colour test. Slanted-edge squares where the eSFR was calculated on 57 regions of interest (ROI) in the MATLAB Image Processing Toolbox (MathWorks, Natick, MA, USA); the results, calculated from the ROI Nr. 5, represent the sharpness at the image edges, and, from the ROI Nr. 29 the sharpness in the image centres.

The 16 patch colours on the printed eSFR ISO test chart were determined as CIE L*a*b* values, defined by the International Commission on Illumination (CIE) on a Datacolor 800 spectrophotometer to calculate accurate patch colour values [41,42]. A diffuse white reflection standard of $8^{\circ}$ measurement field angle geometry was used when the patch was illuminated by a D65 light. The converted colour values of each patch were used as the reference value for colour accuracy analysis.

All cameras were operated without a flashlight, except for the Canon EOS 800D camera, where a flashlight was used as a third light source illumination option. The colour measurements on the flashlight were performed additionally because this camera is used for the TBP in the FotoFinder system, where a flashlight is applied by default [37]. Camera settings, such as white balance, shutter speed, aperture and ISO, were next all set on auto mode. The focus point, however, was set manually at the image centre. Figure 2 shows the applied test chart booth settings. The distance from the camera to the chart was adjusted regarding the camera lens view angle, to cover the sensor surface by approximately 90 percent. 
During the exposure, a camera was fixed on a tripod to avoid vibration, thereby reducing the production of blurry image results [17]. A black fabric material was used to cover the walls in the testing room, to prevent the influence of ambient or other light sources. Calibrated LED lights were applied to illuminate the chart with colour correlated temperatures (CCT) of $3200 \mathrm{~K}$ and $5500 \mathrm{~K}$ (positioned on either camera side at 45 degree angles, and $2 \mathrm{~m}$ in front of the chart table), to estimate the response of the camera to the typical light conditions, as used in the home or hospital environments [8,9]. The intensity of 750 lux and the evenness of light uniformity across the target area were controlled on the different chart positions by the use of a Gossen Digisky light meter.

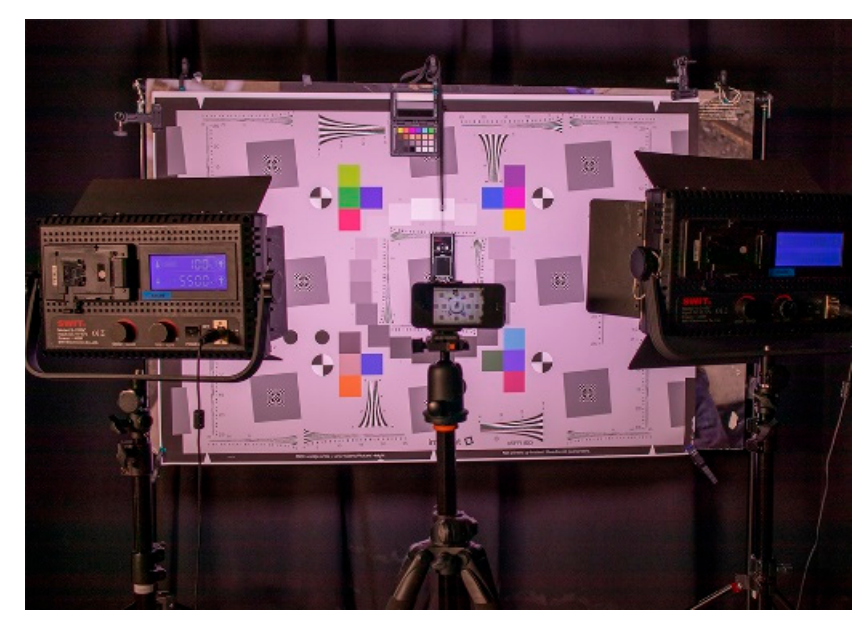

Figure 2. The test chart setup. Two on colour temperature adjustable LED lights are used for a homogeneous illumination. The intensity and the evenness of light uniformity were controlled on a digital light meter.

Our approach was to measure the colour response of a captured image from a reference colour. For this purpose, the test chart was illuminated with lights of colour temperatures $3200 \mathrm{~K}$ and $5500 \mathrm{~K}$. Secondly, the reference colours of the Xrite reference chart [43] were measured as captured through a handheld dermoscopy device and illuminated by the integrated polarised LED light. The colours from the captured images were, after that, evaluated, and compared to the reference value by the CIELAB colour difference $\Delta E$, which is proportional to the colour difference as seen by a human observer $[30,41]$. Dermoscopy images were captured through the DermLite 4 DL handheld dermoscopy device, (Dermlite, 3Gen, Inc., San Juan Capistrano, CA, USA) [44], which was connected by a magnetic ring adapter to a mobile phone and a mirrorless digital camera. The white point of the handheld Dermlite DL4 dermoscope was measured from the lighting profile on a UPRtek MK350 spectrometer. For medical applications, a reference chart with a range of human skin colours is also necessary [30]. Reference skin tone colours in dermoscopy images were captured one-by-one from the Xrite ColorChecker chart, as is shown in Figure 3a. Consequently, colour detection is of great diagnostic importance, and a proper evaluation of colours and their relative distribution is essential for achieving the correct clinical diagnosis of a pigmented skin lesion [27]. During melanoma diagnosis, the dermatologist identifies six different colours: black, dark brown, light brown, blue, grey, red and white $[27,28,45]$. The selection of six reference skin colours of the Digital ColorChecker was based on two facts. First, the melanin and its brown colour determine the basic skin tan, and, at the same time, it represents the essential colour in a dermoscopy image of a skin mole [27]. Identifying multiple shades of brown allows conclusions about the quantity, position and distribution of melanin, which is the primary tool for recognition of melanoma in the earliest stage of the disease. Second, images were imported into the Premiere CC Software (Premiere Pro Version: 14.1, Adobe Inc., San Jose, CA, USA, 2019) for post-processing, to monitor them in a vectorscope view displaying the hue and saturation values of reference skin colours. 
The colour of an individual patch was determined as a numerical value at the image centre as the average of a 51 by 51-pixel area. Exact values of the reference patch colour from the Xrite ColorChecker chart, captured as CIE L*a*b* values, were measured from a Datacolor 800 spectrophotometer.

A micro-test chart was made for image sharpness measured from dermoscopy images. A silver coating layer with a perfectly straight edge line was applied onto a thin glass. The micro test chart with the selected region of interest is shown in Figure 3b. All measurements were performed with the MATLAB Image Processing Toolbox, Adobe Photoshop CC (Photoshop Adobe Photoshop Version: 21.0.3 Adobe Inc., San Jose, CA, USA, 2020) and Adobe Premiere CC software.

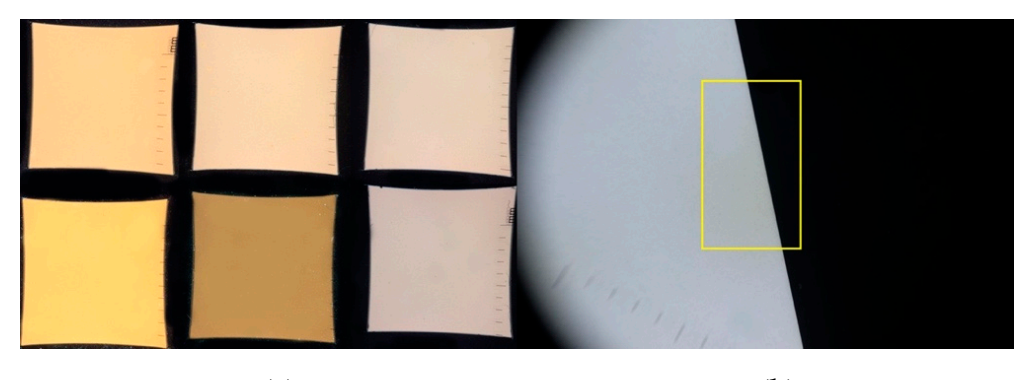

(a)

(b)

Figure 3. (a) Colours of six patches represent human reference skin colours. (b) A slanted edge micro-test chart as applied when sharpness is measured from the target.

\subsection{Image Sharpness and Resolution}

Sharpness determines the amount of detail an imaging system can reproduce, and is a critical image quality factor. Sharpness is defined by the boundaries between zones of different tones. The slanted-edge method of measuring the SFR, as an approximation of the MTF, has become a popular image quality testing method, and multiple international standards have adopted this method, including ISO 12233 and IEEE. The eSFR is based on the frequency response of a slanted edge.

With the 10-90\% rise distance technique, the sharpness of a slanted edge can be determined by the distance of a pixel level between $10 \%$ and $90 \%$ of its intensity transition. The limitation of this method rests in the absence of the possibility to decompose the rise distance of a complete imaging system into the rise distances of its components. This is possible using the frequency domain method, where the frequency is measured in cycles or line pairs per distance. The relative contrast at a given spatial frequency is called MTF, which is the same as the SFR, and is a standard method of measuring sharpness. The shape of the SFR depends on the transfer function of the optical system, sensor technology and, therefore, on the image processing in the camera.

A schematic diagram of the edge-based method is presented in Figure 4. The slanted edges in the eSFR ISO test chart have $5^{\circ}-7^{\circ}$ angles, and the region of interests (ROI) was represented by the rectangle with a slanted edge passing through the short sides, as is illustrated in Figure 4a. The edge angle in a vertical ROI was estimated with a linear regression on the row-to-row edge locations by taking a one-dimensional derivative of the edge gradient in each row. The ROI pixels were then projected onto the horizontal axis. The four-times oversampling was used for minimising anti-aliasing filter problems. The result is a one-dimensional edge spread function (ESF), as presented in Figure $4 \mathrm{~b}$. The approximation of the derivation of the ESF was realised with the finite-difference filter, and, after applying a Hamming window, we were able to calculate the discrete Fourier transform (DFT). Finally, the MTF presented in Figure 4c was estimated as the normalised value of the complex modulus of a discrete Fourier transform. MTF is the Fourier transform of the impulse response that is the derivative of the edge response. The MTF of the sampled image system is a product of the MTF's system components, and is a trustworthy measure for image resolution and sharpness. Spatial frequency is measured in cycles or line pairs per distance, and is the measurement used to determine how much detail a camera can reproduce. The authors used different units for spatial frequency, for example, 
cycles/pixel, cycles/mm and line widths/picture height (LW/PH). The last choice is appropriate when the digital sensor sizes vary widely from small in camera phones to large for full-frame professional cameras. In [46], the author has argued that the MTF50 value of the frequency response correlates well with the perceived sharpness.

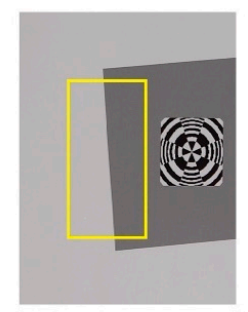

(a)
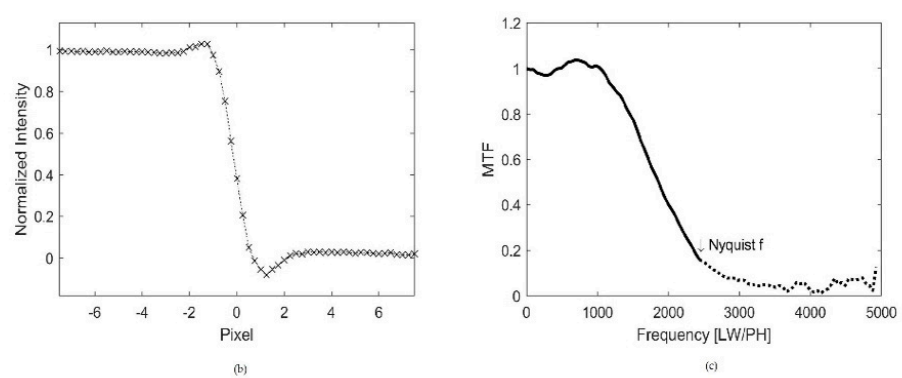

Figure 4. Illustration of slanted edge-based modulation transfer function (MTF) estimation process: (a) selection of a region of interest (ROI), (b) normalised edge spread function (ESF) and (c) estimated MTF.

The MTF50 is the spatial frequency where contrast drops to half (50 percent) of its low-frequency value [47]. Similarly, MTF50P is the spatial frequency where contrast drops to half of its peak value. The authors used both parameters for comparing the sharpness. MTF50P is better than MTF50 for highly sharpened images. Over-sharpening or under-sharpening is the degree to which an image is sharpened relative to a standard sharpening model. Image sharpening is realised mostly with image processing camera software. We calculated the over/under-sharpening degrees following the Imatest recommendations [40]:

$$
\begin{gathered}
\text { Oversharpening }=100 \cdot\left(\operatorname{MTF}\left(f_{\text {eql }}\right)-1\right)(\%) \\
\text { where } f_{\text {eql }}=\left\{\begin{array}{ll}
0.3 \cdot f_{\text {Nyq }} & \text { for MTF50 } \geq 0.2 \text { cycles } / \text { pixel } \\
0.6 \cdot M T F 50 & \text { for MTF50 }<0.2 \text { cycles } / \text { pixel }
\end{array} .\right.
\end{gathered}
$$

When Oversharpening $<0$ the image is under sharpened and Undersharpening $=-$ Oversharpening.

\subsection{Colour Reproduction}

Colour reproduction is considered to be one of the most significant image quality parameters of a digital camera. Even slight deviations in colours captured in photographic images from actual colours can be critical in medical photography, especially in the field of dermatology [23]. The majority of human viewers prefer enhanced images to an image with an exact colour representation [31]. To satisfy viewers' desires, some parameters, like saturation, are often modified by camera manufacturers, to increase the intensity of image colours to make final results more "pleasant". Therefore, the camera manufacturers characterise their sensor technologies to determine the best final image colour accuracy [30]. There is always a difference between "true colours" and colours captured during the photographic process [18]. This colour difference that occurs because of the reproduction quality can be measured.

The International Commission on Illumination (CIE) has defined a system that classifies colour according to the human visual system [30]. Colour differences can be expressed in any space, but they make practical sense only for more uniform spaces where the resulting numbers can be associated with what the eye perceives. The CIE RGB (R-Red, G-Green, B-Blue) and CIE XYZ (X matches to luminance, while $X$ and $Z$ give colour information) were the first defined colour spaces. CIE $X Y Z$ is derived from CIE RGB, where $X Y Z$ are the extrapolation of RGB to avoid negative numbers called tristimulus values [41]. These are device-dependent colour spaces, where the values are dependent on the specific sensitivity function of the imaging system. With the device-dependent model, it is challenging to relate differences between colour with the perception on such colour differences by a human [32]. CIE proposed two other colour models, CIE L*a*b* and CIE $L^{*} u^{*} v^{*}$, which are device-independent models. In the CIE $L^{*} a^{*} b^{*}$ uniform colour space, the perceived luminance $L^{*}$ defines lightness in colour, 
where $\mathrm{a}^{*}$ represents colour relation in a red-green, and $\mathrm{b}^{*}$ represents colour relation in yellow-blue in the chromaticity coordinates [16].

A standardised test chart with known colour patches and their CIE L*a* ${ }^{*}$ values is used to capture images. The CIELAB colour space is based on human vision, and allows the computation of a colour difference. The colour accuracy of an individual colour patch from the test chart is then evaluated by the CIELAB colour difference between the encoded colour in the image and the colour reference data. The difference between two colours, colour distance $\Delta E_{a b}^{*}$, is calculated as the geometric difference between two colour points $a$ and $b$ in the CIELAB three-dimensional colour space as follows [42]:

$$
\Delta E_{a b}^{*}=\sqrt{\left(\Delta L^{*}\right)^{2}+\left(\Delta a^{*}\right)^{2}+\left(\Delta b^{*}\right)^{2}},
$$

where an individual colour is defined with the lightness $\left(L^{*}\right)$ and the hue $\left(a^{*}\right.$ and $\left.b^{*}\right)$ coordinates. The chroma $\Delta C^{*}{ }_{a b}$ difference and the lightness difference $\Delta L^{*}$ between two colours can be determined:

$$
\begin{gathered}
C_{a b}^{*}=\sqrt{\left(a^{*}\right)^{2}+\left(b^{*}\right)^{2}}, \\
\Delta C_{a b}^{*}=C_{a b 1}^{*}-C_{a b 2^{\prime}}^{*} \\
\Delta L^{*}=L_{1}^{*}-L_{2}^{*} .
\end{gathered}
$$

The mean colour distance $\Delta E_{\text {mean }}$ is then calculated from 16 (six patches are used for dermoscopy images) colour patches $\Delta E_{a b}^{*}$ values.

\section{Results}

Relevant camera parameters, i.e., sharpness, resolution and colour reproduction, were measured, and are presented in this section. The algorithms for measuring sharpness and colour reproduction were applied to the same standardised chart targets. Sharpness results are explained in the next two subsections and summarised in two Tables.

\subsection{Results of Sharpness and Resolution Measurements}

The captured images from the cameras were processed by a programme which was written by using the functions from the MATLAB Image Processing Toolbox. To analyse the sharpness, we plotted four captured images using two plots: a plot with a normalised intensity profile across a sharp edge, and another plot with a corresponding MTF. From the first plot, we read a key sharpness measure, known as the edge rise distance (10-90\%), shown in pixels. It should be noted that the smaller the measured distances in pixels are, the sharper is the captured edge profile. Other parameters examined included both overshoot and undershoot, which characterise the degree of over/under sharpening of the captured image. From the SFR presented on the second plot, we read MTF50 as an important measurement result, which corresponds similarly to perceived image sharpness. Higher values of the $\mathrm{LW} / \mathrm{PH}$ correspond to a sharper image. If additional sharpening is applied by a camera processor's software, the MTF50 and MTF50P are not equal in value.

\subsubsection{Cameras' Sharpness Results for the Imatest ${ }^{\circledR}$ eSFR Test Chart}

Photos to measure the sharpness of all seven cameras were taken twice at different temperatures of the LED lights that were used for illumination, namely $3200 \mathrm{~K}$ and $5500 \mathrm{~K}$. The sharpness measurements 10-90\%, MTF50, and over/under sharpness in Table 2 below come from two areas (the rectangle just above the higher left of the chart-ROI5 and the rectangle in the centre of the chart-ROI29) of the ISO 12233 image, illustrated by red rectangles in the image presented in Figure 1. We made three shots when using the Canon EOS 800D camera. On the third shot, we used the Canon Speedlite 430EX II flash for lighting. The results are summarised in Table 2. 
Table 2. Cameras' sharpness results for the Imatest ${ }^{\circledR}$ eSFR test chart.

\begin{tabular}{|c|c|c|c|c|c|c|c|c|}
\hline \multirow{2}{*}{ Camera } & \multirow{2}{*}{$\begin{array}{l}\text { Nyquist f } \\
\text { (LW/PH) }\end{array}$} & \multirow{2}{*}{$\mathrm{CT}[\mathrm{K}]$} & \multicolumn{3}{|c|}{ ROI 5} & \multicolumn{3}{|c|}{ ROI 29} \\
\hline & & & $\begin{array}{c}10-90 \% \\
\text { (px) }\end{array}$ & $\begin{array}{c}\text { MTF50 } \\
\text { (LW/PH) }\end{array}$ & $\begin{array}{c}\text { O/U Sharp } \\
(\%)\end{array}$ & $\begin{array}{c}10-90 \% \\
\text { (px) }\end{array}$ & $\begin{array}{c}\text { MTF50 } \\
\text { (LW/PH) }\end{array}$ & $\begin{array}{c}\text { O/U Sharp } \\
(\%)\end{array}$ \\
\hline \multirow{2}{*}{ Iphone 5s } & \multirow{2}{*}{2448} & 3200 & 1.2 & 1862 & 3.7 & 0.8 & 2322 & 14.2 \\
\hline & & 5500 & 1.2 & 1946 & -3.3 & 0.9 & 2362 & 16.4 \\
\hline \multirow{2}{*}{ Iphone 7} & \multirow{2}{*}{3024} & 3200 & 2.5 & 1260 & -25.9 & 1.3 & 2139 & 0.8 \\
\hline & & 5500 & 2.4 & 1367 & -21.1 & 1.3 & 2305 & 7.0 \\
\hline \multirow{2}{*}{ Iphone XR } & \multirow{2}{*}{3024} & 3200 & 1.1 & 2090 & 6.3 & 0.9 & 2331 & 13.6 \\
\hline & & 5500 & 1.2 & 1927 & 20.1 & 0.9 & 2654 & 36.9 \\
\hline \multirow{2}{*}{ Samsung S10 } & \multirow{2}{*}{3024} & 3200 & 0.9 & 2512 & 61.4 & 0.6 & 2982 & 67.3 \\
\hline & & 5500 & 0.7 & 2693 & 87.5 & 0.5 & 3052 & 111.9 \\
\hline \multirow{2}{*}{ Huawei P10 } & \multirow{2}{*}{2976} & 3200 & 1.2 & 1998 & 27.1 & 1 & 2058 & 36.8 \\
\hline & & 5500 & 1.2 & 1932 & 19.4 & 1 & 2116 & 38.4 \\
\hline \multirow{2}{*}{$\begin{array}{c}\text { Canon EOS } \\
\text { M10 }\end{array}$} & \multirow{2}{*}{3458} & 3200 & 3 & 1383 & -28.1 & 1.1 & 2437 & 40.2 \\
\hline & & 5500 & 3 & 1388 & -27.2 & 1.1 & 2440 & 45.4 \\
\hline \multirow{3}{*}{$\begin{array}{c}\text { Canon EOS } \\
\text { 800D } \\
\text { (FotoFinder) }\end{array}$} & \multirow{3}{*}{4000} & 3200 & 1.9 & 1907 & 3.8 & 1.2 & 2543 & 32.8 \\
\hline & & 5500 & 1.9 & 1856 & 5.8 & 1.2 & 2474 & 38.0 \\
\hline & & Flash & 1.7 & 2101 & 10.6 & 1.3 & 2415 & 27.0 \\
\hline
\end{tabular}

The graphs for analysing the sharpness of all captured images of the eSFR test chart with the iPhone 7 camera are presented in Figure 5. On the left are plots of the edge profile curves, and on the right side are corresponding MTF plots. The difference in sharpness parameters of the object in the left higher corner (ROI5), and the object in the image centre (ROI29) can be read from the plots. In the case of this camera, illumination with different lights had a negligible impact on the sharpness of the captured images. From the test results, we can estimate that the iPhone 7 camera software used to generate the JPG format does not force image sharpening processes. This is not the case with the other cameras we tested, especially the Samsung S10 and Huawei P10, where we measured a significant degree of over-sharpness, as shown in Table 1. All cameras tested showed better sharpness in the centre than at the edges of the images.
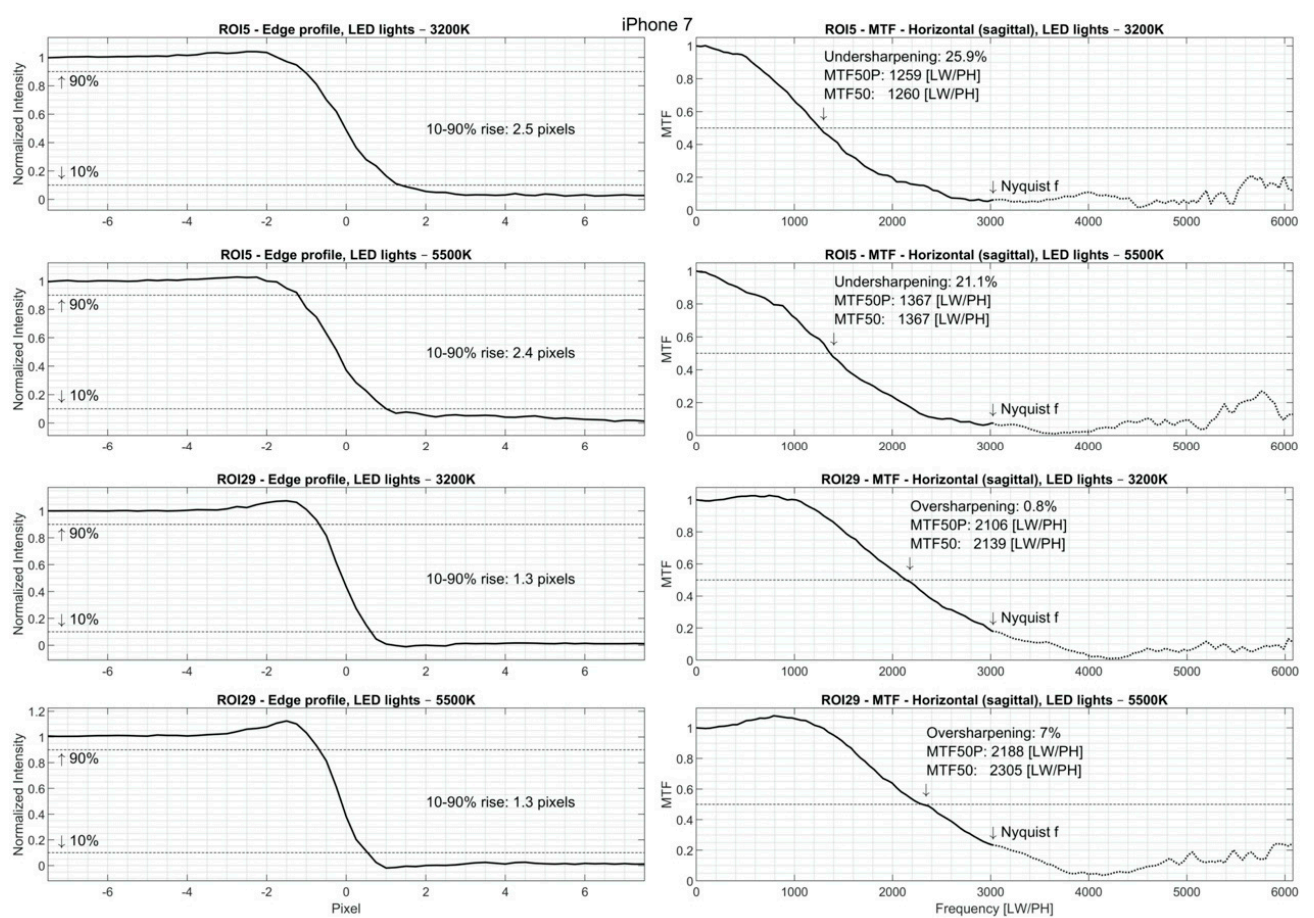

Figure 5. Edge profile curves and plots with the corresponding MTF for the higher left corner of the image (ROI5) and for the centre of the image (ROI29). Images were captured with the iPhone7 camera under different illuminations with calibrated LED lights (3200 K and $5500 \mathrm{~K}$ ). 


\subsubsection{Cameras + DermLite 4 DL Handheld Dermoscope Sharpness Results}

The tested dermoscopy images were captured with the DermLite 4 DL handheld dermoscope attached to a smartphone or a mirrorless digital camera lens. For image sharpness analysis, we used a micro-test chart where a thin silver coating layer was applied onto a glass plate, which provided a slanted edge that was perfectly straight.

To understand the image quality of the optical system Camera + DermLite 4 DL handheld dermoscope accurately, it was critical to combine the effects of each component to determine how the system will affect sharpness. Every component-the phone lens, the camera, and the handheld dermoscope- has an MTF value, and by multiplying each MTF, we were able to arrive at a value of a system MTF. In Figure 6, we present the graphs that analyse the sharpness of the iPhone 7 camera and DermLite 4 DL handheld dermoscope. The shape of the MTF shows a significant loss of sharpness. The MTF50 decreased to the value $1479 \mathrm{LW} / \mathrm{PH}$, edge rise distance (10-90\%) was 5.8 pixels, and we calculated under sharpening of 18.7 .
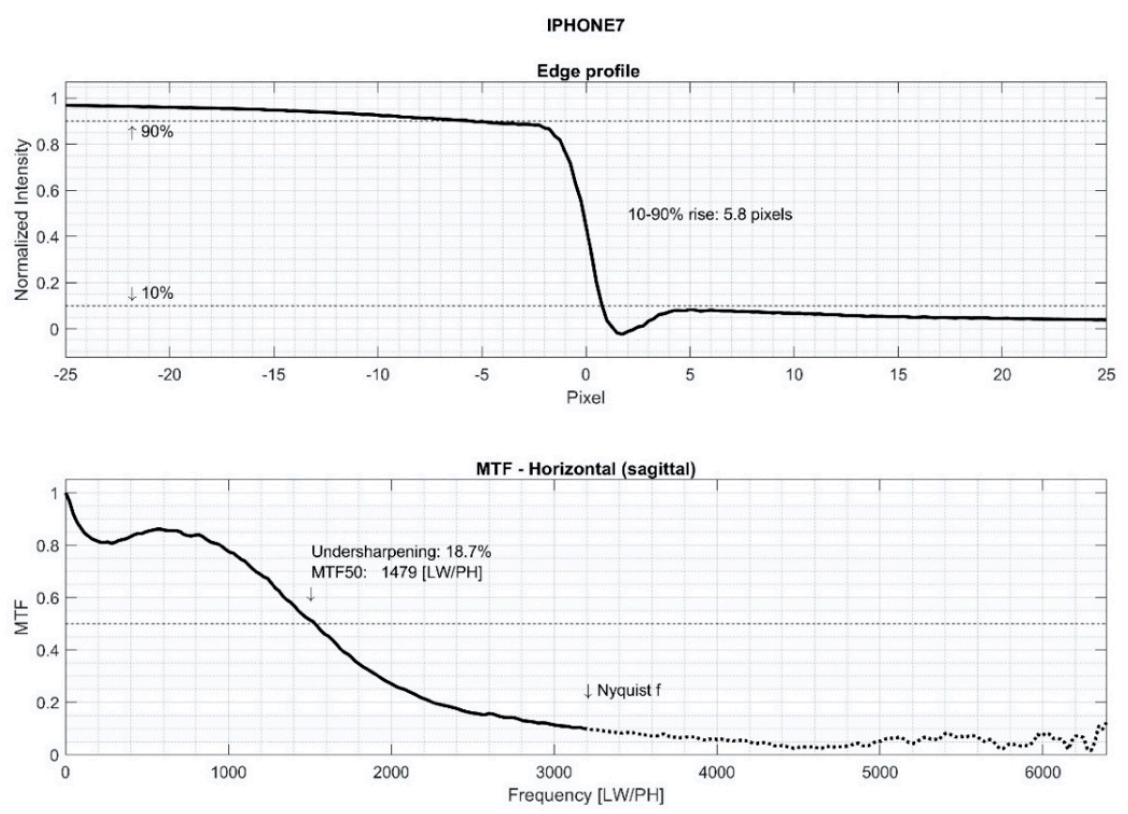

Figure 6. Sharpness results for iPhone 7 camera with DermLite 4 DL dermoscope.

The results from the other five cameras that we used in conjunction with a mounted handheld dermoscopy device are summarised in Table 3. The sharpness measurements obtained with the state-of-the-art digital dermoscopy system FotoFinder with a micro camera Medicam 1000s are presented in the last row of Table 3. Among all devices tested, the Medicam 1000s camera yielded the lowest values regarding image resolution. The numerical value indicates that this camera is not superior compared to the other cameras, at least if overall quality is evaluated only on sharpness and resolution. However, the Medicam 1000s is supported by an optical zoom lens with 20 to 400 times magnification, that compensates for the lower resolution of the camera sensor.

Table 3. Cameras with DermLite 4 DL dermoscope sharpness results-macro test chart.

\begin{tabular}{ccccc}
\hline Camera & Nyquist f (LW/PH) & $\mathbf{1 0 - 9 0 \% ~ ( P i x e l s ) ~}$ & MTF50 (LW/PH) & Over/Under Sharpness (\%) \\
\hline Iphone 5s & 2448 & 10 & 1116 & -36.6 \\
Iphone 7 & 3024 & 5.8 & 1479 & -18.7 \\
Iphone XR & 3024 & 15.7 & 1048 & -25.9 \\
Samsung S10 & 3024 & 16.3 & 1399 & 12.2 \\
Huawei P10 & 2976 & 8.6 & 796 & -38.1 \\
Canon EOS M10 & 3456 & 13.5 & 1399 & -34.5 \\
Medicam 1000s (FotoFinder) & 1080 & 1.9 & 519 & -9.2 \\
\hline
\end{tabular}




\subsubsection{Influence of Applied Sharpening of Dermoscopy Image in Melanoma Diagnosis}

The image sharpening process refers to any enhancement technique which concentrates on the edges and the fine details of an image. Sharpening operations are manifest through the SFR. With high sharpening, image noise and image sampling artefacts can be amplified along with the signal content. From the results in Table 2, it is evident that the tested cameras have image sharpening algorithms with different levels of sharpness.

Two cropped images of the slanted edge presented in Figure 7e,f were captured with a Canon EOS M10 camera. In Figure 7e, the image is captured with a low sharpening level and a gradual transition is visible from the bright area to the dark area. The high level of the sharpness was set by capturing the image on Figure 7f, where the transition from the bright area to the dark area is more contrasting, and the artefacts and noise are visible. We wondered how these artefacts influence the assessment of dermatologists when observing dermoscopy images.

In clinical evaluation, high sharpening of dermoscopy images emphasises artefacts (pieces of the dead epidermis, hair follicles, dust particles, air bubbles ... ), as well as colour and structural changes (Figure 7a,b), especially on additional magnification in partial image evaluation (Figures $7 \mathrm{c}$ and $8 \mathrm{~d}$ ). The changes that result from image sharpening can, therefore, reduce the capability to recognise characteristic signs for melanoma, especially on larger magnification and in cases when the dermoscopy procedure is performed by inexperienced dermatologists.
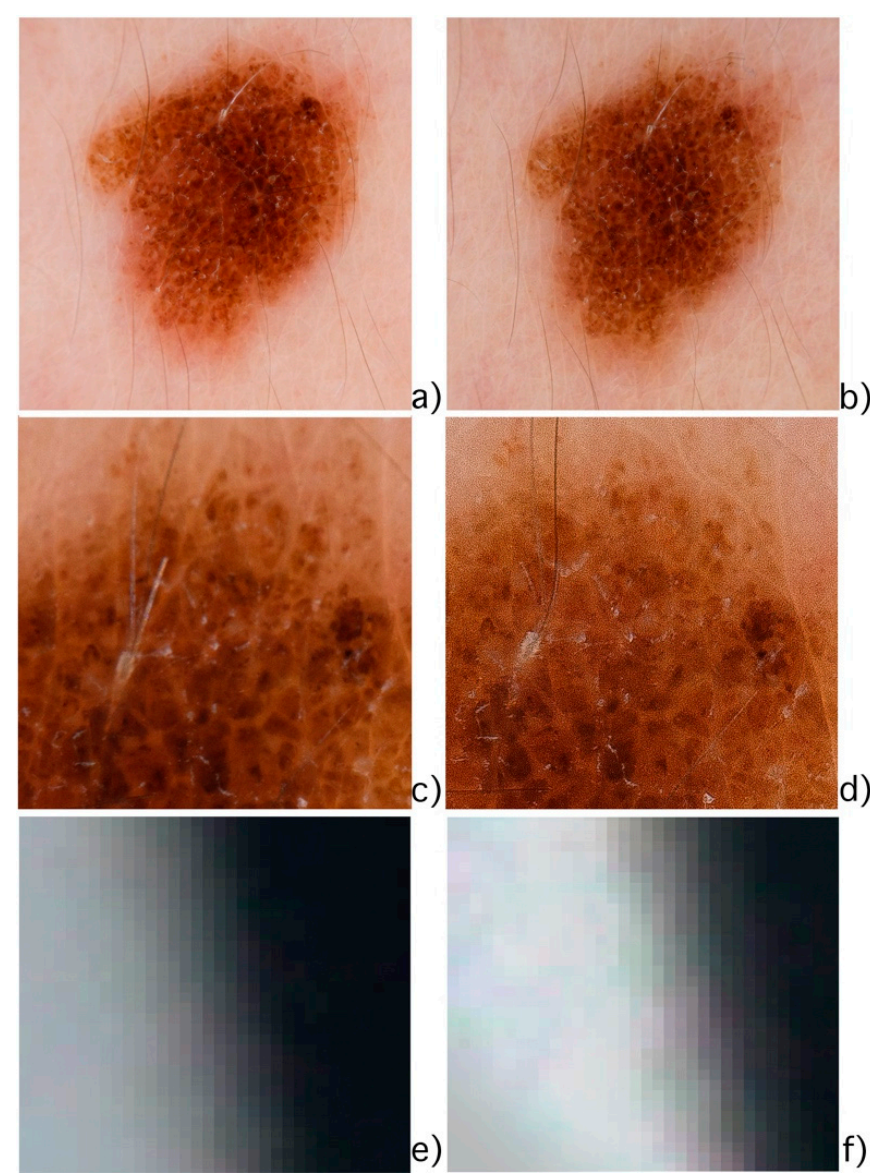

Figure 7. Influence of sharpening in a mole dermoscopy image captured with a Canon EOS M10 camera with a DermLite 4 DL dermoscope (a) with a low sharpening level (b) with a high sharpening level, (c) a low sharpening level in partial image evaluation, (d) a high sharpening level in partial image evaluation, (e) cropped image of slanted edge in low sharpening level, and (f) cropped image of slanted edge in high sharpening level. 


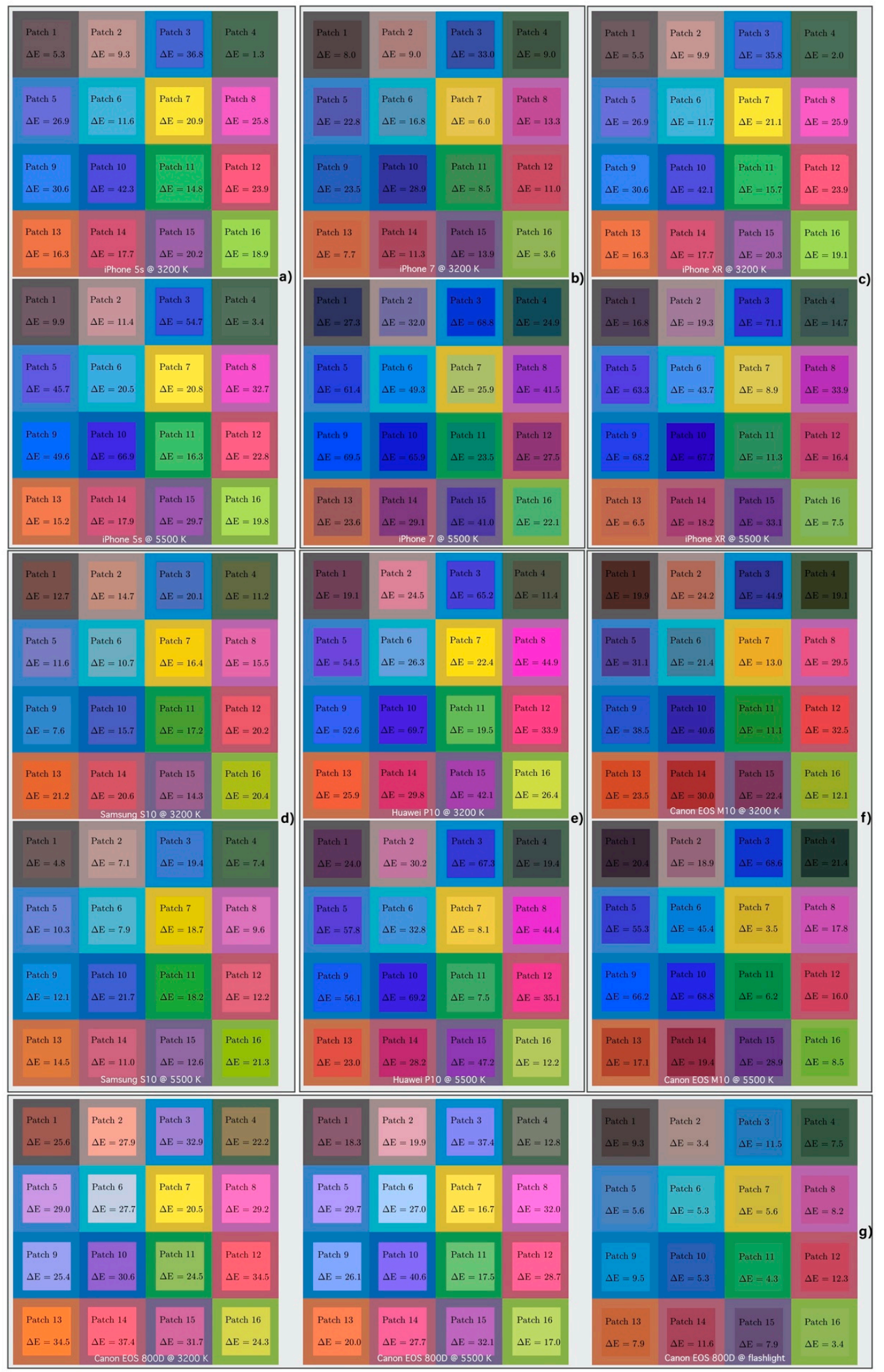

Figure 8. The colour difference between captured and reference colours from the test chart is measured and derived as the CIELAB $\Delta E$ colour distance. Diagrams for illuminance light source of $3200 \mathrm{~K}$ and $5500 \mathrm{~K}$ colour temperatures are presented, and for the Canon EOS 800D with the flashlight in the last row. Diagrams of captured colours are shown for the following devices: iPhone 5 s (a), iPhone 7 (b), iPhone XR (c), Samsung S10 (d), Huawei P10 (e), Canon EOS M10 (f) and Canon EOS 800D (g). 


\subsection{Results of Colour Measurements}

Our approach was both to measure the colour error distance between captured image colours and reference colours printed on a standard eSFR chart target, as well as the colour accuracy for the dermoscopy-captured images using the six skin colours from the Xrite chart. We measured colour responses from images captured on the eSFR chart and illuminated under three different lights; namely, $3200 \mathrm{~K}$ and $5500 \mathrm{~K}$ LED and a flashlight. Secondly, the colour response and the error distances were calculated from colours of images captured from the reference colours' patches on the Xrite chart through a handheld dermoscopy device with integrated polarised LED light source. Data from a camera image are available in the sRGB colour space, and have been encoded to CIELAB values. The results of the colour measurements are provided in the next two subsections and summarised in two Tables.

\subsubsection{Image Colours}

There is a difference between colours in captured images and true colours. Colour accuracy is given as the CIELAB $\Delta E^{*}{ }_{a b}$ colour error difference value, calculated with Equation (1) between the encoded image patch colour and the reference colour. The charts presented in Figure 8 show the colour difference between captured images and their corresponding reference colour (what we might term "true" colour) from the test chart. The variation, or the difference in colours, is reflected as their $\Delta E$ values, measured from spectroradiometer colour data when the chart was illuminated with lights of colour temperatures $3200 \mathrm{~K}$ and $5500 \mathrm{~K}$. We measured 16 different colour patches. The last row represents colour charts and differences in values using the Canon EOS 800D camera. A flashlight colour chart is included because this camera is used in the FotoFinder system for TBP, where a flashlight is applied by default. The individual colour patches in the chart are numbered, as shown in Figure 1.

Figure 8 illustrates colour differences for each colour patch, and gives calculated $\Delta E$ colour distance values regarding the reference values. Cameras' responses to individual colours are quite different. For example, the maximal colour distances $\Delta E_{\max }$ measured on an image captured by the Canon EOS 800 camera were detected for the red colour $\Delta E_{\max }(34.5 @ 3200 \mathrm{~K})$, light brown $\Delta E_{\max }(27.9 @ 3200 \mathrm{~K})$ and, dark blue $\Delta E_{\max }(40.6 @ 3200 \mathrm{~K})$. Concerning the images captured by the iPhone 7 , on the other hand, the colour blue yielded the highest colour difference, and was measured with maximum $\Delta E_{\max }$ estimated on the patch no. $9 \Delta E_{\max }(69.5 @ 5500 \mathrm{~K})$. Small colour errors were recognised for brown, green and yellow. The best test results regarding reference values were achieved when a flashlight was used, and measured from an image captured by the Canon EOS 800D. The maximum distance was measured for the red colour, calculated from patch no. 12, with a colour difference of $\Delta E_{\max }(12.3 @$ @flash). However, when comparing the results in Table 4, we can see that the Canon EOS 800D had no advantages over the other models when the chart was illuminated with lights of colour temperatures $3200 \mathrm{~K}$ and $5500 \mathrm{~K}$. Therefore, it is to be expected that other cameras in the test will render colours more precisely if the target is illuminated by an external xenon flashlight, because it has a higher value of colour rendering index (CRI) than any LED light. Applying a flashlight to a digital camera is easy as most cameras already integrate a built-in flashlight, or external flashlights can be used additionally. The light power of a built-in flash LED light of smartphones is insufficient for total-body imaging, and the support of external illumination is mandatory. Smartphone photographing with an external flashlight is possible with wireless triggering over a Bluetooth connection, or with a separate radio adapter.

Results on images captured by the smartphone Samsung S10, by both light source temperatures, were close to the reference values. An interesting finding is that, when using a Huawei P10 smartphone, capturing images of the colour blue yielded responses with significant errors, i.e., with vast distances to the reference, where the maximum value was measured for the colour no. $10 \Delta E_{\max }(69.2 @ 3200 \mathrm{~K})$. These findings led the authors to conclude that this particular camera is manufactured in a fashion to yield a more saturated blue colour. Similar results were obtained from images captured by the iPhone 5s, iPhone XR and Canon M10 camera, where blue colour tones seem to be the least accurate. The mean colour distances $\Delta E_{\text {mean }}$ over 16 colours measured from the charts, minimum $\Delta E_{\text {min }}$ and maximum $\Delta E_{\max }$ colour distance for tested cameras and used illuminants are summarised in Table 4 . 
The results in Table 4 show that the best $\Delta E_{\text {mean }}$ in auto mode with $\Delta E_{\text {mean }}=13.1 @ 5500 \mathrm{~K}$ (and $\Delta E_{\text {mean }}$ $=13.1 @ 3200 \mathrm{~K})$ distance was achieved by the Samung S10 camera, and the iPhone 7 with $\Delta E_{\text {mean }}=$ $39.6 @ 5500 \mathrm{~K}$ was the highest measured mean value. The iPhone 5 responds with the best minimal colour error $\Delta E_{\text {mean }}=1.3 @ 3200 \mathrm{~K}$.

Table 4. Colour accuracy is given with colour distances as mean value $\Delta E_{\text {mean }}$, minimum $\Delta E_{\text {min }}$ and maximum $\Delta E_{\max }$, calculated on 16 colours from the test chart.

\begin{tabular}{ccccc}
\hline Camera & CT (K) & $\Delta E_{\text {mean }}$ & $\Delta E_{\text {min }}$ & $\Delta E_{\text {max }}$ \\
\hline \multirow{2}{*}{ iPhone 5s } & 3200 & 20.2 & 1.3 & 42.3 \\
& 5500 & 27.3 & 3.4 & 66.9 \\
\hline \multirow{2}{*}{ iPhone 7 } & 3200 & 14.1 & 3.6 & 33 \\
& 5500 & 39.6 & 22.1 & 69.5 \\
\hline \multirow{2}{*}{ iPhone XR } & 3200 & 20.3 & 2.0 & 42.1 \\
& 5500 & 31.3 & 6.5 & 71.1 \\
\hline \multirow{2}{*}{ Samsung S10 } & 3200 & 15.6 & 7.6 & 21.2 \\
& 5500 & 13.1 & 4.8 & 21.7 \\
\hline \multirow{2}{*}{ Huawei P10 } & 3200 & 21.6 & 13.3 & 21.7 \\
& 5500 & 35.2 & 7.5 & 69.2 \\
\hline \multirow{2}{*}{ Canon EOS M10 } & 3200 & 25.9 & 11.1 & 37.4 \\
& 5500 & 30.2 & 3.5 & 68.8 \\
\hline \multirow{2}{*}{ Canon EOS 800D } & 3200 & 28.6 & 20.5 & 37.4 \\
& 5500 & 25.2 & 12.8 & 40.6 \\
& Flash & 7.4 & 3.4 & 12.3 \\
\hline
\end{tabular}

\subsubsection{Colours in the Dermoscopy Image}

Dermoscopy images were captured through the DermLite 4 DL handheld dermoscope attached to a smartphone or digital camera lens. Image colour analysis was performed on a set of six skin-colour patches from the $X$-Rite test chart. Due to the small dimension of the dermoscope viewing field (approximately $20 \mathrm{~mm}$ in diameter), each colour patch was captured and measured separately.

In Figure 9, colours and their differences concerning reference colours for auto mode camera setting from dermoscopy captured images are presented on the map in two layers: the captured colour is in the foreground, and the reference colour is in the background. The resulting impression on the dermoscopy captured images indicates that the images are generally overexposed, and, in general, the $\Delta E$ colour difference response is relatively high as compared to the reference values. The $\Delta E$ colour distance calculated from a captured image regarding the reference colour is shown in Table 5. The first column in Table 5 identifies the name of the device used. The second column lists the mean colour difference $\Delta E_{\text {mean }}$ on six different tested colours. The third and fourth columns present the maximum $\Delta E_{\text {max }}$ and minimum $\Delta E_{\text {min }}$ difference values. The results in Table 5 show that the smallest $\Delta E_{\text {mean }}=$ 15.0 distance was achieved evaluating images captured by the Huawei P10 camera, with the highest colour distance of $\Delta E_{\max }=22.7$. Therefore, our measuring revealed that low error responses were achieved when images were captured either by the iPhone XR or the Canon EOS M10 camera. 
Table 5. Colour reproduction accuracy is given for auto and manual mode camera setting as mean $\Delta E_{\text {mean }}$ colour distance, minimal $\Delta E_{\min }$ and maximal $\Delta E_{\text {max }}$ value regarding the six skin tone reference colours.

\begin{tabular}{ccccccc}
\hline & \multicolumn{3}{c}{ Auto Mode } & \multicolumn{3}{c}{ Manual Mode } \\
\hline Camera & $\boldsymbol{\Delta} \boldsymbol{E}_{\text {mean }}$ & $\boldsymbol{\Delta} \boldsymbol{E}_{\text {min }}$ & $\boldsymbol{\Delta} \boldsymbol{E}_{\text {max }}$ & $\boldsymbol{\Delta} \boldsymbol{E}_{\text {mean }}$ & $\boldsymbol{\Delta} \boldsymbol{E}_{\text {min }}$ & $\boldsymbol{\Delta} \boldsymbol{E}_{\text {max }}$ \\
\hline iPhone 5s & 32.1 & 15.4 & 43.1 & 9.7 & 6.4 & 12.8 \\
iPhone 7 & 31.0 & 12.5 & 62.7 & 9.1 & 5.2 & 11.7 \\
iPhone XR & 20.6 & 12.5 & 25.3 & 9.6 & 5.1 & 12.9 \\
Samsung S10 & 33.3 & 19.1 & 42.6 & 11.1 & 5.7 & 16.1 \\
Huawei P10 & 15.0 & 4.5 & 22.7 & 9.3 & 6.0 & 15.3 \\
Canon EOS M10 & 20.5 & 2.8 & 43.4 & 11.3 & 7.8 & 14.4 \\
Medicam 1000s & 45.7 & 26.2 & 64.7 & - & - & - \\
\hline
\end{tabular}

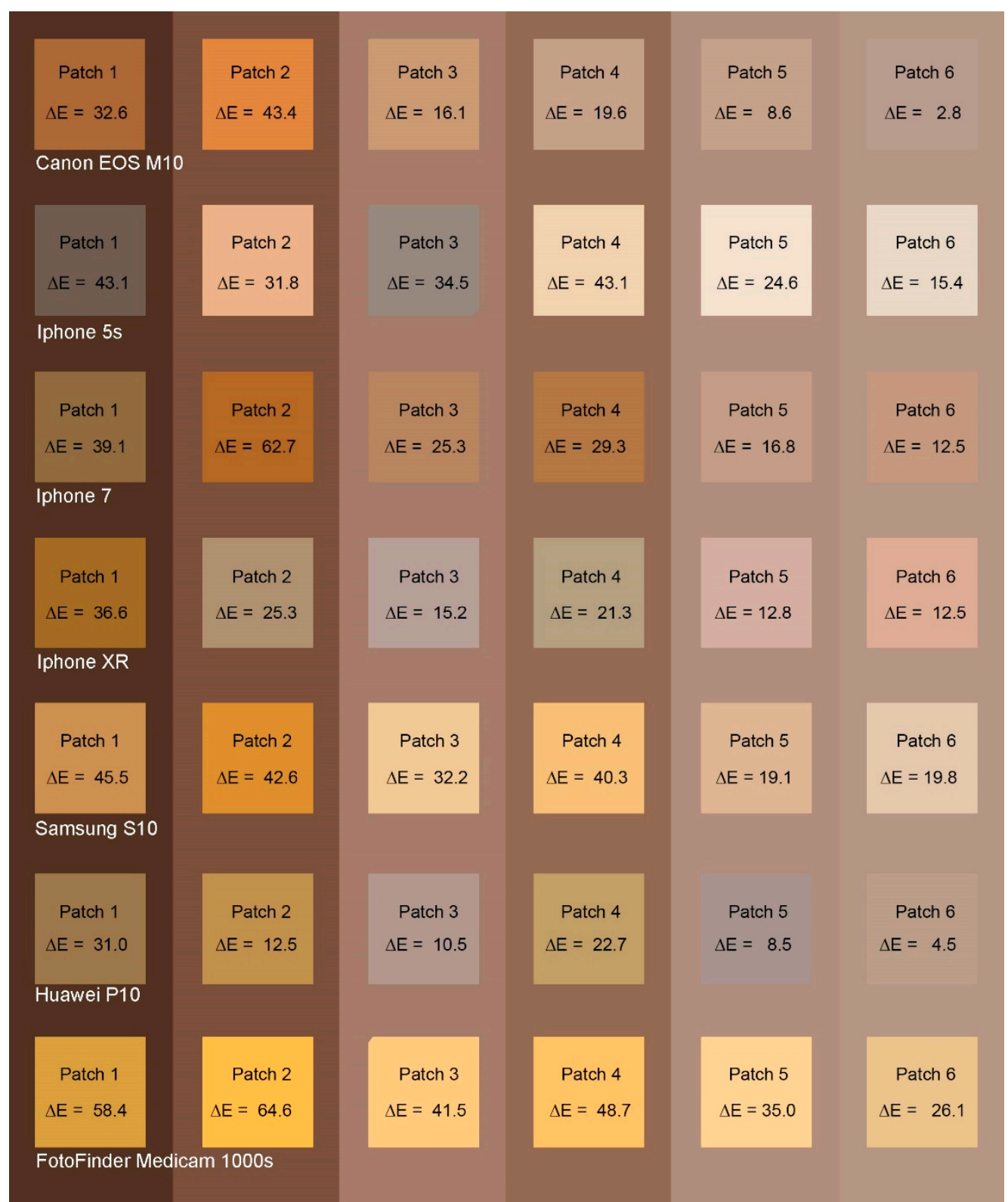

Figure 9. The colour presentation of dermoscopy images about reference colours for auto mode camera setting. The CIELAB $\triangle E$ colour distance of skin tone colour patches are set forth. 
The $\Delta E$ colour distance values for calibrated cameras (manual mode setting) is shown in Table 5 . Columns five, six and seven present the mean $\Delta E_{\text {mean }}$, minimum $\Delta E_{\min }$ and maximum $\Delta E_{\max }$ on measured skin colours after application of a device calibration. The result shows a significant improvement in colour constancy, where colours are much closer to the range of human eye detection capability. Practical interpretations of $\Delta E$ colour errors can be found in the works of Hardeberg et al. [48] and Aborado et al. [49]. Colour errors in video and printing standards are expected between 5 and 6, between 6 and 10 the quality is still sufficient, and above ten as insufficient [48]. The mean colour error value $\Delta E_{\text {mean }}$ for the iPhone and Huawei P10 camera models fulfil the criteria of Hardeberg et al. [48]; however, results for the Canon EOS M10 and the Samsung S10 exceeded the recommended value of Hardeberg slightly. The parameters of the Fotofinder Medicam 1000s were measured only in a default camera setting, as the manufacturer does not allow any corrections of camera parameters by the user.

The $\Delta E_{\text {mean }}$ colour difference values measured from the FotoFinder's Medicam 1000s images in-camera default mode are high. Consequently, the resulting images appear overexposed, and the colours are so visually different from the reference colour that they are evident to the naked eye. Uncorrected images were imported into the Premiere CC Software to monitor them in a vectorscope view. A vectorscope monitor displays the colour values in the image. The angle of the pixel represents the hue, and the distance out from the centre represents the amount of saturation.

Pixels at the centre of the vectorscope are grey, and the colour hue starts with red in the top left, and, rotating clockwise, transitions to magenta, to blue, to cyan, to green, to yellow, and, finally, back to red. Figure 10a illustrates reference colours, presented in the vectorscope with dots. Dots of reference colours are aligned precisely on the skin tone line, between the Red $(\mathrm{R})$ colour channel and the Yellow $(\mathrm{Y})$ colour channel. Figure 10b show dots presenting colours from the captured image on the Medicam 1000s. The position of dots is now shifted into the yellow section, but on the line. The uncorrected microscopic image of a mole captured on the same camera is presented in Figure 11a. The colour correction was applied to improve the colours on the image, as shown in Figure 11b. The distribution of colours in the corrected image is shifted toward the reference line, as shown in the vectorscope in Figure 11b.

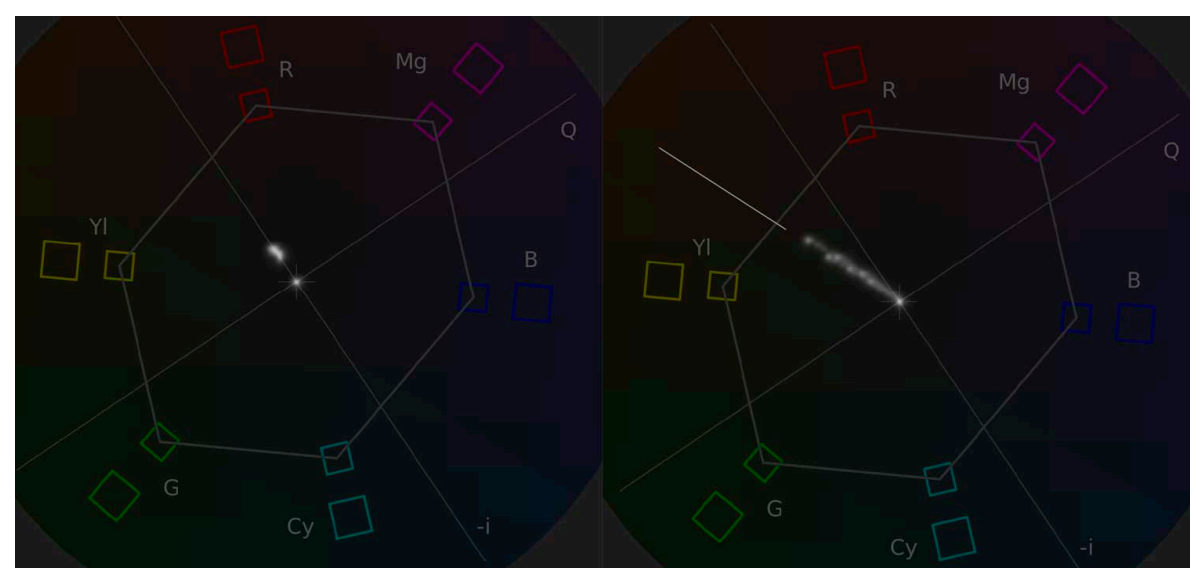

(a)

(b)

Figure 10. Colours are presented in the vectorscope as dots. (a) Dots are aligned on the "skin tone line" presenting the reference value of the skin tone colours, and (b) dots presenting colours in the image if the reference colours were captured on the Medicam 1000s camera. 


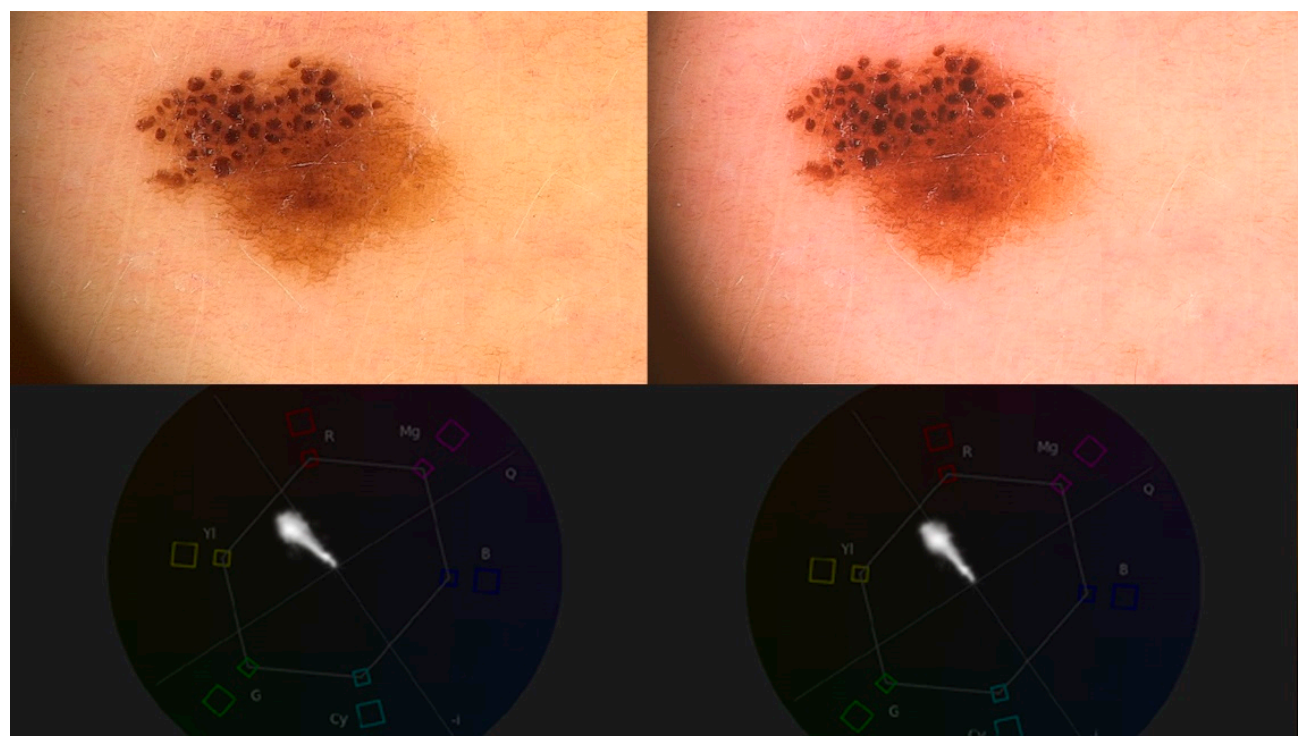

(a)

(b)

Figure 11. (a) Dermoscopy image of a skin mole and the corresponding dispersion of colours is shown in the vectorscope. (b) The colour corrected image with the vectorscope colour dispersion. The skin colour is more natural and closer to what the dermatologist is used to seeing with his naked eye.

For essential subjective evaluation, we verified the effectiveness of the camera calibration process on a set of dermoscopy images. We compared images that were captured with the same camera, which works in automatic or manual mode. Dermoscopy images of the same lesion were captured on the Fotofinder Medicam 1000s, Canon EOS M10, and iPhone 7 cameras. The results in Table 5 show that the most optimal result in manual mode with $\Delta E_{\text {mean }}=9.1$ was achieved by the iPhone 7 and the Canon M10 $\Delta E_{\text {mean }}=11.3$ was the highest measured colour error after calibration was applied. Figure 12 shows examples of dermoscopy images before and after calibration. Figure 12a shows a dermoscopy image captured with a Fotofinder Medicam 1000s, and Figure 12b shows an image after a colour correction performed with a software tool. Figure 12c,e show images captured on the Canon EOS M10 and iPhone 7 in automatic camera mode, and Figure 12d,f show modified images captured after camera calibration. The colour differences in the image captured by the Fotofinder Medicam 1000s before and after the processing are minimal if the photos are compared side by side. Colour and structural changes could not be detected subjectively. The significant colour variations can be detected in the images taken with the camera of the mobile phone and digital camera, before and after calibration. In Figure 12c,e, we immediately detected an irregular skin colour in the surrounding area of the mole (almost grey), which disturbs the dermoscopy evaluation of the structures at the margins of the mole. The colour of the mole in Figure 12c,e is subjectively darker (brown), which, according to dermoscopic rules, means that the observed melanocytic lesion (including melanoma) is less profound and, therefore, less dangerous. In manually captured images, Figure $12 \mathrm{~d}, \mathrm{f}$, the colours are subjectively more correct, a more enhanced sharpness enables easier identification of mole structures. Based on the measurement results and subjective perception, we concluded that images (Figure 12d,f) acquired manually on calibrated cameras are more comparable with images acquired on the digital Fotofinder Medicam 1000s (Figure 12b). 


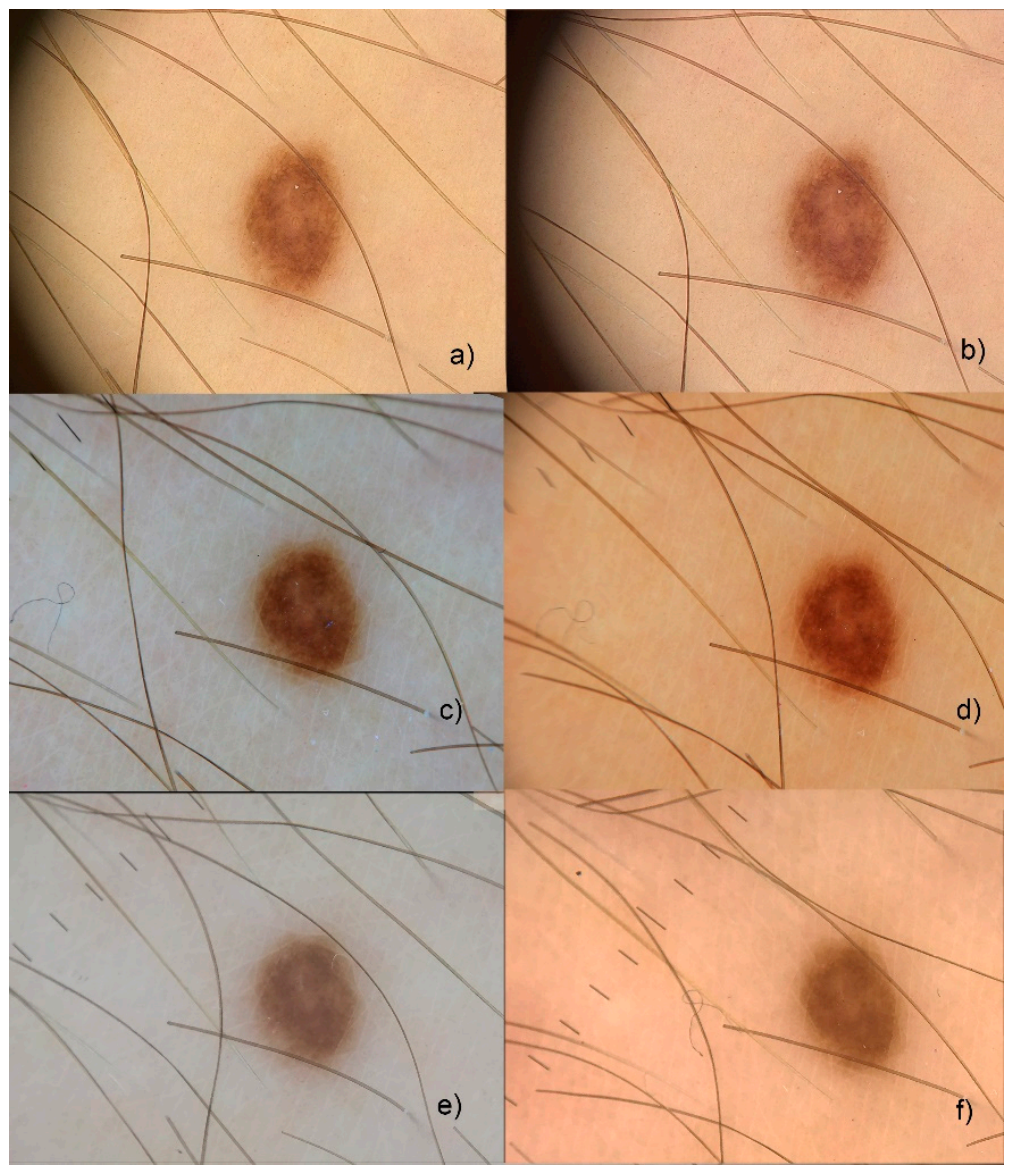

Figure 12. Skin mole dermoscopy images captured on (a) Fotofinder Medicam 1000s (factory settings), (b) Fotofinder Medicam 1000s with colour correction, (c) Canon EOS 10M in auto mode, (d) Canon EOS M10 manual colour white balance (CWB) setting, (e) iPhone 7 in auto mode, and (f) iPhone 7 manual CWB setting.

\subsection{Algorithm for Optimal Capturing of Dermoscopy Images in Melanoma Diagnostics}

An algorithm for image capture, assessment and display using automatic or manual mode is presented in Figure 13. After preparing a setup that consists of of a digital camera or smartphone coupled with a handheld dermoscope, the region of interest (ROI) on a skin surface is determined. In the next the step, the user could select between automatic or manual camera mode.

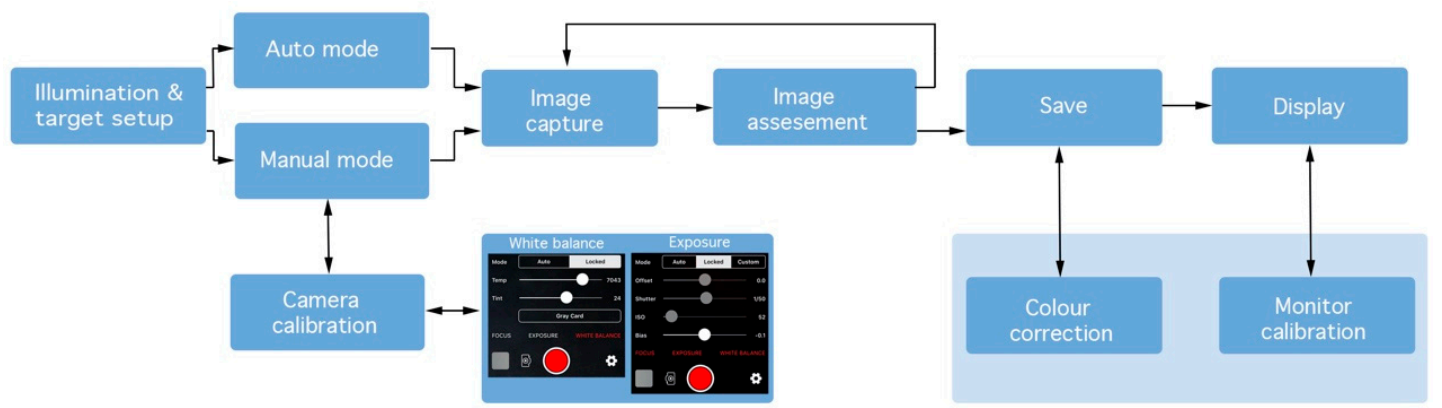

Figure 13. Algorithm for image capturing, assessing and displaying in automatic or manual camera mode.

In the automatic mode, all camera parameters are adjusted automatically; in the case of too low quality in the assessment step, the user has to recapture a photo with a correction of the ROI on the skin. 
In the manual mode, a camera must be calibrated first. The calibration requires the adjustments of two camera parameters, colour white balance (CWB) and shutter speed. The most effective way to perform colour calibration is adjusting the CWB in the camera settings to match the illumination light profile. To avoid under- or overexposed images, the exposure time is locked on a middle grey colour patch value (neutral 8) of a ColorChecker Classic target. The calibration takes a few minutes of user time, and remains valid while the light condition remains unchanged. In the next step, images are captured and evaluated on other distortions, such as motion or defocus blur. A successfully captured image is saved in JPG or in RAW format. Images can be displayed directly on the camera's or smartphone's screen, or on a large computer monitor.

\subsection{Displays}

Medical displays used in melanoma diagnostics need to achieve a minimum performance level to ensure that all details in images are visible to the dermatologists [50]. For realistic colour presentation, monitors must be calibrated regularly.

A calibration method ensures that the colour output from a monitor matches a predefined standard, such as the DICOM, sRGB or Adobe RGB colour space [51]. Monitors can be calibrated with some freely available software tools, and the built-in calibration tools within Windows and macOS. The hardware monitor calibrator is the most accurate method to calibrate displays. A calibrator detects the colours emitted by a screen and creates a custom software ICC profile. The use of ICC profiles for achieving a good colour reproduction across devices [52] is already a well-established practice in other fields of Medicine.

\subsection{Assesement and Recommendations for Image Captruring Devices}

The following recommendations are suggested to evaluate the suitability of a mobile device camera for dermatological photography:

- A larger sensor is preferable to a smaller one (Table 1 ) as it yields a higher-quality image. A camera's sensor determines the number of pixels in the photographs it produces. With between 8 and 14 million pixels, the dermatologist's photography needs are covered. More pixels would only be needed to maintain good resolution or size when cropping, or if TBP is applied.

- Digital cameras can save JPEG files in different sizes with varying degrees of compression. Use the highest quality JPEG setting (i.e., with the lowest compression of image information) possible to keep the detail.

- The camera should allow manual settings and white balance adjustment. Ensure the imaging device has an appropriate white balance setting to allow the most accurate colour rendition.

- For displaying an image preview on a camera/mobile device monitor, the following criteria should be considered: monitor resolution (and size) $>1920 \times 1080 \mathrm{px}(14 \mathrm{~cm})$ or more for a smartphone, and $>1.000 \mathrm{k}$ dots $(14 \mathrm{~cm})$ or more for a digital camera.

- Handheld dermoscopes must be supported with an adapter that ensures a firm fit and optimum position of the lens system in front of the camera to ensure the best image sharpness. Several handheld dermoscopes already produce universal attachments allowing for their compatibility with any smartphone. Lens size should be at least $20 \mathrm{~mm}$ in diameter, and a larger lens size is preferable. Some dermoscopes are already supported by a smartphone application for capturing and image management. It would be helpful to have integrated the assistance for automatic sharpness recognition [17].

Our results (Tables 2-4) show that we have selected from all tested devices the iPhone 7 as a suitable device for capturing dermoscopic images. We made the decision based on measurements showing good sharpness of dermoscopic images and solid color reproduction with small mean absolute error in manual mode. This result is due mainly to the less aggressive sharpening algorithm implemented in 
this device. The device provides many manual settings. With the correct setting of the image capture parameters, the reproduction quality of skin colours is improved dramatically.

\section{Discussion}

Currently, dermatologists want to identify melanoma at the earliest stage of the disease, when it is, in most cases, similar to a benign pigment mole in the naked eye examination. Dermoscopy improves diagnosis accuracy significantly, even in early stages of melanoma [52-57]. To detect melanoma at an early stage, the most subtle colour shades (especially brown) and structures need to be identified by dermoscopy, often only in a small part of the lesion [58-60]. Colour assessment is essential for the clinical diagnosis of skin cancers [6,25-29]. Therefore, dermatologists need a dermoscopy device able to provide an image with authentic colours and sharply defined tiny structures in the lesion, as well as an image that offers the same good quality throughout the whole dermoscopic field, including at magnification $[6,8]$. Routine handheld dermoscopy is performed increasingly with convenient and affordable systems consisting of handheld dermoscope optics combined with a mobile phone. For people with high levels of pigment moles and, consequently, high risk of melanoma, sovereign diagnosis of melanoma is still possible only with digital dermoscopy and TBP, where the routine use is still limited by the high purchase price and time-consuming diagnostics [61,62]. Routine handheld dermoscopy is performed increasingly with convenient and affordable systems consisting of handheld dermoscope optics combined with a mobile phone.

The handheld dermoscope, when connected to a mobile phone camera or a digital camera, generates a simple, digital dermoscopy system which can provide a good substitute for a highly-priced digital dermoscopy device. Unfortunately, combining camera and different optical systems is not always an optimal solution, since images captured in this fashion may well contain several additional artefacts which could impact the overall image quality negatively [8].

In our study, dermoscopy images were captured using the Dermlite handheld dermoscopy device attached to either a smartphone or a high-resolution camera. The images were then compared with the results obtained on a digital dermoscopy system, which is considered as the gold standard.

The results show that the sharpness reduces slightly in the centre of an image if a handheld dermoscope is attached to a smartphone or digital camera. Moreover, the sharpness decreases significantly towards the edges of the image. Another concern is the applied sharpening of the images by a camera signal processor, which has a significant impact on the SFR of a camera system. It increases the contrast on lower spatial frequencies, which makes the image virtually sharper to a human observer [20]. Based on the results of our study, almost all tested cameras use a certain degree of additional sharpening.

The overly sharp image emphasises artefacts (pieces of the dead epidermis, hair follicles, dust particles), making it difficult for dermoscopic diagnostics, especially for inexperienced dermatologists. However, furthermore, images are captured frequently by dermatologists in automatic camera mode, although that digital imaging device already allows a manual setting of different camera parameters. In the camera settings, the user can select the most appropriate individual sharpening level.

The TBP method is being applied increasingly for skin cancer monitoring, and is used to survey existing and identify new or changing lesions, where high image resolution and sharpness are significant features $[11,24,38]$. State-of-the-art digital dermoscopy systems integrate a high-resolution digital camera for TBP [37]. In this setting, a serial of horizontally oriented photographs is captured until the patient's entire body surface is covered. The weakness of the TBP is that the automated capturing procedure is hugely time-consuming, both for patients and physicians, where the patient has to be photographed in various positions to cover the full body [10]. The TBP implementation in our test applies a built-in DSLR camera with a resolution of $6000 \times 4000$ pixels [37]. The camera is horizontally oriented, and four images must be captured to cover each patient position [6]. However, the number of photos and time for TBP can be reduced significantly by rotating the camera through 90 degrees to capture images vertically instead of horizontally. Upgrading an existing camera with a higher 
resolution camera (i.e., $9504 \times 6336$ pixels) can replace four horizontally taken images with a single shot image of vertical orientation. That reduces the total number of shots, maintains high image resolution and shortens the time it takes to capture images of a full body significantly.

Our results show that images captured with uncalibrated mobile devices or digital cameras may cause notable colour variations. Cameras have difficulty adjusting the appropriate parameters (inappropriate colours and brightness) when operating in automatic mode, especially when using light from a handheld dermoscope. Therefore, the cameras should always be calibrated to minimise colour differences between different imaging devices and the illuminant characteristic. However, almost all digital cameras also allow users to adjust the parameters manually. One of the simplest but effective methods is to adjust the colour white balance (CWB) manually based on the given light conditions. The correct camera exposure time is determined using the reference middle grey background to ensure the correct illumination of the images. This two-step camera calibration procedure improves the colour accuracy of both clinical and dermoscopy images significantly. The results show that colours are much closer to the range of a human eye's detection capability, with a colour error that is smaller, as recommended in Hardeberg [48].

However, the colour accuracy of dermoscopy images can be improved further by using custom colour profiles (ICC). These can be created for a known exposure profile, but can only be generated for RAW images. However, to create an ICC profile, we need software that recognises the differences between standardised colour values and those captured. Such software is already available to professional photographers as a standalone commercial version, or as a third-party software plugin.

Using ICC profiles would also be one option for colour correction of dermoscopy images. This method first requires a calibrated target to be able to create an appropriate ICC profile. However, to date, the smallest available target ColorChecker Classic Nano, with its size $24 \mathrm{~mm} \times 40 \mathrm{~mm}$, does not match the field of view of existing handheld dermoscopes. Some improvements could be addressed for future work. A specific target should be developed for hand dermoscopes that could improve the colour calibration process as well as a test tool. It can be used both as a calibration tool, as well for testing new devices (i.e., a handheld dermoscopy device coupled with a camera). The target will be based on an attachable glass with a printed test target. The target will allow measuring colour response, resolution and sharpness, and can be combined with colour correction software as a simple camera calibration tool.

\section{Conclusions}

Photographing with a non-standardised device like a smartphone or a digital camera to document the skin changes is becoming increasingly popular among dermatologists. Due to the rapid development of new devices, diagnostic methods and applications based on a recognition of skin cancer through captured images, it is necessary to ensure credible methodologies and standards for assessing camera systems.

In this paper, the authors discussed measurement results that we made to assess the image quality of digital imaging systems for melanoma detection. In particular, we measured such variables as sharpness, resolution and colour accuracy. The standard methods, based on the eSFR of a slanted edge, were applied for measuring the sharpness and resolution of a camera system. Colours of known reference values were captured from the test target to evaluate colour error as a CIELAB $\Delta E_{a b}^{*}$ colour difference.

The results of our measurements yielded two significant findings. First, that currently on-the-market cameras, when operating in the auto mode, render overly saturated colours. Second, the applied sharpening level, especially estimated for images captured by some of the smartphone cameras tested, is set too high. Both the auto mode feature and applied sharpening technology are incorporated into the camera software by default by the manufacturers, to produce images that have the most "eye-appeal" for the broadest population of end-users.

A two-step camera calibration procedure was performed to improve colour accuracy on dermoscopy images. The applied method is simple and does not take too much effort and dermatologist's time. In the first step, the white balance on the camera was adjusted to match the light source of a dermoscope, and in 
the second, the proper exposure time was estimated regarding a reference middle grey value, to avoid under- or overexposed images. A manually adjusted camera setting gives a significant improvement in the colour accuracy where colours are much closer to the range of the human eye's detection capability.

Author Contributions: Conceptualisation, A.D., M.M. and B.D.; methodology, A.D., B.D. and M.G.; software, M.G. and B.D.; validation, B.D., A.D. and M.G.; writing-original draft preparation, B.D.; writing-review and editing, B.D.; M.G.; M.M. and A.D.; visualisation, B.D., A.D. and M.G.; supervision, A.D. and B.D. All authors have read and agreed to the published version of the manuscript.

Funding: This research received no external funding.

Conflicts of Interest: The authors declare no conflict of interest.

\section{References}

1. Garbe, C.; Amaral, T.; Peris, K.; Hauschild, A.; Arenberger, P.; Bastholt, L.; Bataille, V.; Grob, J.; Del Marmol, V.; Dréno, B.; et al. European consensus-based interdisciplinary guideline for melanoma. Part 2 Treatment-Update. Eur. J. Cancer 2019, 126, 159-177. [CrossRef] [PubMed]

2. Conforti, C.; Giuffrida, R.; Vezzoni, R.; Resende, F.S.; di Meo, N.; Zalaudek, I. Dermoscopy and the experienced clinicians. Int. J. Dermatol. 2020, 59, 16-22. [CrossRef]

3. Marghoob, A.A.; Usatine, R.; Jaimes, N. Dermoscopy for the family physician. Am. Fam. Physician 2013, 88, 441-450. [PubMed]

4. International Society for Digital Imaging of the Skin (ISDIS) Project. Available online: https://isdis.org/isicproject/ (accessed on 27 February 2020).

5. Marek, A.J.; Chu, E.Y.; Ming, M.E.; Khan, Z.A.; Kovarik, C.L. Impact of a smartphone application on skin self-examination rates in patients who are new to total body photography: A randomized controlled trial. J. Am. Acad. Dermatol. 2018, 79, 564-567. [CrossRef] [PubMed]

6. Finneane, A.; Curiel-Lewandrowski, C.; Wimberley, G.; Caffery, L.; Katragadda, C.; Halpern, A.; Marghoob, A.; Malvehy, J.; Abraham, I.; Kittler, H.; et al. Proposed technical guidelines for the acquisition of clinical images of skin-related conditions. JAMA Dermatol. 2017, 153, 453-457. [CrossRef]

7. Pagliarello, C.; Stanganelli, I.; Fabrizi, G.; Feliciani, C.; Di Nuzzo, S. Digital Dermoscopy Monitoring: Is it Time to Define a Quality Standard? Acta Derm. Venereol. 2017, 97, 864-865. [CrossRef]

8. Quigley, E.A.; Tokay, B.A.; Jewell, S.T.; Marchetti, M.A.; Halpern, A.C. Technology and technique standards for camera-acquired digital dermatologic images: A systematic review. JAMA Dermatol. 2015, 151, 883-890. [CrossRef] [PubMed]

9. Katragadda, C.; Finnane, A.; Soyer, H.P.; Marghoob, A.A.; Halpern, A.; Malvehy, J.; Kittler, H.; Hofmann-Wellenhof, R.; Da Silva, D.; Abraham, I.; et al. Technique standards for skin lesion imaging: A Delphi consensus statement. JAMA Dermatol. 2017, 153, 207-213. [CrossRef]

10. Hibler, B.P.; Qi, Q.; Rossi, A.M. Current state of imaging in dermatology. Semin. Cutan. Med. Surg. 2016, 35, 2-8. [CrossRef]

11. Lee, K.J.; Soyer, H.P. Future developments in teledermoscopy and total body photography. Int. J. Dermatol. Venereol. 2019, 2, 15-18.

12. Asaid, R.; Boyce, G.; Padmasekara, G. Use of a smartphone for monitoring dermatological lesions compared to clinical photography. J. Mob. Technol. Med. 2012, 1, 16-18. [CrossRef]

13. McKoy, K.; Antoniotti, N.M.; Armstrong, A.; Bashshur, R.; Bernard, J.; Bernstein, D.; Burdick, A.; Edison, K.; Goldyne, M.; Kovarik, C.; et al. Practice Guidelines for Teledermatology. Telemed. E-Health 2016, 22, 981-990. [CrossRef] [PubMed]

14. Commissioning, P.C. Quality Standards for Teledermatology Using 'Store and Forward' Images. 2013. Available online: https://www.bad.org.uk/shared/get-file.ashx?itemtype=document\&id=794 (accessed on 4 April 2020).

15. ISO 12233:2014-Photography: Electronic still Picture Imaging-Resolution and Spatial Frequency Responses. Available online: https:/ww.iso.org/obp/ui/\#iso:std:iso:12233:ed-2:v1:en (accessed on 10 June 2019).

16. Rosado, L.; Vasconcelos, M.J.; Castro, R.; Tavares, J.M. From dermoscopy to mobile teledermatology. In Dermoscopy Image Analysis; CRC Press: Boca Raton, FL, USA, 2015. 
17. Alves, J.; Moreira, D.; Alves, P.; Rosado, L.; Vasconcelos, M.J.M. Automatic Focus Assessment on Dermoscopic Images Acquired with Smartphones. Sensors 2019, 19, 4957. [CrossRef] [PubMed]

18. Fricke, D.; Denker, E.; Heratizadeh, A.; Werfel, T.; Wollweber, M.; Roth, B. Non-Contact Dermatoscope with Ultra-Bright Light Source and Liquid Lens-Based Autofocus Function. Appl. Sci. 2019, 9, 2177. [CrossRef]

19. Burns, P.D. Slanted edge MTF for digital camera and scanner analysis. In Is and Ts Pics Conference; Society for Imaging Science \& Technology: Springfield, VA, USA, 2000; pp. 135-138.

20. Artmann, U. Image quality assessment using the dead leaves target: Experience with the latest approach and further investigations. In Digital Photography XI; International Society for Optics and Photonics: San Francisco, CA, USA, 2015; Volume 9404, p. 94040J.

21. Chazallet, F.; Glasser, J. Theoretical bases and measurement of the MTF of integrated image sensors. In Image Quality: An Overview; International Society for Optics and Photonics: San Francisco, CA, USA, 1985; Volume 549, pp. 131-144.

22. Masaoka, K. Accuracy and precision of edge-based modulation transfer function measurement for sampled imaging systems. IEEE Access 2018, 6, 41079-41086. [CrossRef]

23. Schneider, S.L.; Kohli, I.; Hamzavi, I.H.; Council, M.L.; Rossi, A.M.; Ozog, D.M. Emerging imaging technologies in dermatology: Part I: Applications and limitations. J. Am. Acad. Dermatol. 2019, 80, 1114-1119. [CrossRef]

24. Dahlén Gyllencreutz, J.; Johansson Backman, E.; Terstappen, K.; Paoli, J. Teledermoscopy images acquired in primary health care and hospital settings-A comparative study of image quality. J. Eur. Acad. Dermatol. Venereol. 2018, 32, 1038-1043.

25. Weismann, K.; Lorentzen, H.F. Dermoscopic color perspective. Arch. Dermatol. 2006, 142, 1250. [CrossRef]

26. Duteil, L.; Roussel, K.; Bahadoran, P. Skin Color and Pigmentation. In Agache's Measuring the Skin; Humbert, P., Fanian, F., Maibach, H., Agache, P., Eds.; Springer: Cham, Switzerland, 2017.

27. Kittler, H.; Rosendahl, C.; Cameron, A.; Tschandl, P. Dermatoscopy: An Algorithmic Method Based on Pattern Analysis; Facultas Verlags: Vienna, Austria, 2011.

28. Marghoob, A.; Braun, R. (Eds.) An Atlas of Dermoscopy; CRC Press: Boca Raton, FL, USA, 2012.

29. Madooei, A.; Drew, M.S. Incorporating colour information for computer-aided diagnosis of melanoma from dermoscopy images: A retrospective survey and critical analysis. Int. J. Biomed. Imaging 2016. [CrossRef]

30. Penczek, J.; Boynton, P.A.; Splett, J.D. Color error in the digital camera image capture process. J. Digit. Imaging 2014, 27, 182-191. [CrossRef]

31. Wueller, D. Cell Phone Camera. Electron. Imaging 2017, 37-45. [CrossRef]

32. Haeghen, Y.V.; Naeyaert, J.M.A.D.; Lemahieu, I.; Philips, W. An imaging system with calibrated color image acquisition for use in dermatology. IEEE Trans. Med. Imaging 2000, 19, 722-730. [CrossRef] [PubMed]

33. Grana, C.; Pellacani, G.; Seidenari, S. Practical color calibration for dermoscopy, applied to a digital epiluminescence microscope. Ski. Res. Technol. 2005, 11, 242-247. [CrossRef] [PubMed]

34. Quintana, J.; Garcia, R.; Neumann, L. A novel method for color correction in epiluminescence microscopy. Comput. Med. Imaging Graph. 2011, 35, 646-652. [CrossRef] [PubMed]

35. Iyatomi, H.; Celebi, M.E.; Schaefer, G.; Tanaka, M. Automated color calibration method for dermoscopy images. Comput. Med. Imaging Graph. 2011, 35, 89-98. [CrossRef]

36. Barata, C.; Celebi, M.E.; Marques, J.S. Improving dermoscopy image classification using color constancy. IEEE J. Biomed. Health Inform. 2014, 19, 1146-1152.

37. FotoFinder. Available online: http://www.fotofinder.de/ (accessed on 22 September 2019).

38. Micali, G.; Lacarrubba, F. Dermatoscopy: Instrumental Update. Dermatol. Clin. 2018, 36, 345-348. [CrossRef]

39. Imatest-ISO 122233:2017 TEST CHARTS. Available online: http://www.imatest.com/solutions/iso-12233/ (accessed on 10 June 2019).

40. Imatest-Sharpness. Available online: http://www.imatest.com/docs/sharpness/ (accessed on 10 June 2019).

41. Pascale, D. RGB Coordinates of the Macbeth ColorChecker; The BabelColor Company: Montreal, QC, Canada, 2006; Volume 6.

42. Billmeyer, F.W., Jr.; Fairman, H.S. CIE method for calculating tristimulus values. Color Res. Appl. 1987, 12, 27-36. [CrossRef]

43. X-Rite ColorChecker. Available online: http://xritephoto.com/colorchecker-classic (accessed on 22 September 2019).

44. Dermlite. Available online: https://apps.apple.com/app/dermlite/id840789465 (accessed on 12 October 2019). 
45. Sáez, A.; Acha, B.; Serrano, A.; Serrano, C. Statistical detection of colors in dermoscopic images with a texton-based estimation of probabilities. IEEE J. Biomed. Health Inform. 2018, 23, 560-569.

46. Koren, N. The Imatest program: Comparing cameras with different amount of sharpening. In Digital Photography II; International Society for Optics and Photonics: San Francisco, CA, USA, 2006; Volume 6069, p. 60690L.

47. Artmann, U. Linearization and normalization in spatial frequency response measurement. Electron. Imaging 2016, 2016, 1-6. [CrossRef]

48. Hardeberg, J.Y. Acquisition and Reproduction of Color Images: Colorimetric and Multispectral Approaches; Universal-Publisher: Irvine, CA, USA, 2001.

49. Abrardo, A.; Alparone, L.; Cappellini, V.; Prosperi, A. Color Constancy from Multispectral Images. In Proceedings of the International Conference on Image Processing IEEE, Kobe, Japan, 24-28 October 1999.

50. Visualization of Medical Content on Color Display Systems. International Color Consortium. Available online: www.color.org/whitepapers/ICC_White_Paper46-Medical_Photography_Guidelines.pdf (accessed on 7 April 2020).

51. Marghoob, A.A. Standards in dermatologic imaging. JAMA Dermatol. 2015, 151, 819-821. [CrossRef] [PubMed]

52. Silverstein, L.; Hashmi, S.; Lang, K.; Krupinski, E. Paradigm for achieving color-reproduction accuracy in LCDs for medical imaging. J. Soc. Inf. Disp. 2012, 20, 53-62. [CrossRef]

53. Bono, A.; Bartoli, C.; Cascinelli, N.; Lualdi, M.; Maurichi, A.; Moglia, D.; Marchesini, R. Melanoma detection. Dermatology 2002, 205, 362-366. [CrossRef] [PubMed]

54. Bafounta, M.L.; Beauchet, A.; Aegerter, P.; Saiag, P. Is dermoscopy (epiluminescence microscopy) useful for the diagnosis of melanoma? Results of a meta-analysis using techniques adapted to the evaluation of diagnostic tests. Arch. Dermatol. 2001, 137, 1343-1350. [CrossRef] [PubMed]

55. Vestergaard, M.E.; Macaskill, P.H.P.M.; Holt, P.E.; Menzies, S.W. Dermoscopy compared with naked eye examination for the diagnosis of primary melanoma: A meta-analysis of studies performed in a clinical setting. Br. J. Dermatol. 2008, 159, 669-676. [CrossRef]

56. Bowling, J.; Argenziano, G.; Azenha, A.; Bandic, J.; Bergman, R.; Blum, A.; Hofman-Wellenhof, R. Dermoscopy key points: Recommendations from the international dermoscopy society. Dermatology 2007, 214, 3-5. [CrossRef]

57. Holmes, G.A.; Vassantachart, J.M.; Limone, B.A.; Zumwalt, M.; Hirokane, J.; Jacob, S.E. Using Dermoscopy to Identify Melanoma and Improve Diagnostic Discrimination. Fed. Pract. 2018, 35 (Suppl. 4), 39.

58. Borsari, S.; Longo, C.; Ferrari, C.; Benati, E.; Bassoli, S.; Schianchi, S.; Seidenari, S. Dermoscopic island: A new descriptor for thin melanoma. Arch. Dermatol. 2010, 146, 1257-1262. [CrossRef]

59. Carli, P.; De Giorgi, V.; Chiarugi, A.; Nardini, P.; Mannone, F.; Stante, M.; Giannotti, B. Effect of lesion size on the diagnostic performance of dermoscopy in melanoma detection. Dermatology 2003, 206, 292-296. [CrossRef]

60. Silva, V.P.M.D.; Ikino, J.K.; Sens, M.M.; Nunes, D.H.; Di Giunta, G. Dermoscopic features of thin melanomas: A comparative study of melanoma in situ and invasive melanomas smaller than or equal to $1 \mathrm{~mm}$. An. Bras. Dermatol. 2013, 88, 712-717. [CrossRef]

61. Moloney, F.J.; Guitera, P.; Coates, E.; Haass, N.K.; Ho, K.; Khoury, R.; Menzies, S.W. Detection of primary melanoma in individuals at extreme high risk: A prospective 5-year follow-up study. JAMA Dermatol. 2014, 150, 819-827. [CrossRef] [PubMed]

62. Salerni, G.; Carrera, C.; Lovatto, L.; Puig-Butille, J.A.; Badenas, C.; Plana, E.; Malvehy, J. Benefits of total body photography and digital dermatoscopy ("two-step method of digital follow-up") in the early diagnosis of melanoma in patients at high risk for melanoma. J. Am. Acad. Dermatol. 2012, 67, e17-e27. [CrossRef] [PubMed]

(C) 2020 by the authors. Licensee MDPI, Basel, Switzerland. This article is an open access article distributed under the terms and conditions of the Creative Commons Attribution (CC BY) license (http://creativecommons.org/licenses/by/4.0/). 